\title{
The Ph.D. Dilemma in Canada Revisited
}

\author{
MAX VON ZUR-MUEHLEN, Ph.D.**
}

\begin{abstract}
Growth of doctoral studies at Canadian universities in the last two decades has resulted in the more than 1,000 programs that are now offered. Not surprisingly, the output of Ph.D. graduates has increased 6 fold since the early six ties. But during the seventies, an imbalance between the rising supply of Ph.D. 's and the declining demand for them, particularly in higher education, became apparent. This paper traces historical trends in the employment of Canada's Ph.D. holders, and looks at their prospects for the future.

Traditionally, about $65 \%$ of doctoral graduates have entered educational occupations. Today, because of the youthful age structure, there are few retirements or deaths, and hence, the annual replacement demand is for only about 500 Ph.D. 's. But Canadian universities now confer around 2,000 doctorates each year (including returning Canadians from abroad).

Moreover, this imbalance is apt to persist. On the basis of the current enrolment of 13,000, the Ph.D. supply has been projected from 1977-78 to 1981-82 for 45 disciplines. Relating these supply estimates to the likely demand for university teachers reveals a potential surplus in almost every discipline. A cycle of shortage and surplus appears to have developed in some fields. These simulations have been derived from assumptions, which are outlined in two appendices and 26 supporting tables.

In addition, this paper also examines other features of the Ph.D. situation in Canada: a history of the growth of graduate education; variations in the ratio of Ph.D. enrolment to graduates in different disciplines; support programs for doctoral students, and the immigration of university teachers.

The information provides an overview of the many dimensions of the Ph.D. issue.
\end{abstract}

\section{RESUME \\ Le Dilemme du doctorat au Canada, revu -}

La croissance de l'intérêt pour les études au doctorat a été telle au cours des deux dernières décennies, que plus de 1,000 programmes sont présentement offerts dans les universités canadiennes. D'où la constatation que le nombre de détenteurs d'un doctorat soit six fois plus grand depuis les années 60. Toutefois, au cours des années 70 , un déséquilibre s'est manifesté, surtout dans le domaine de l'enseignement supérieur, entre le nombre croissant

* The vicws expressed by the author are his own and not necessarily those of Statistics Canada.

** Institutional and Public Finance Statistics Branch, Statistics Canada. 
de diplômés d'un doctorat d'une part et la demande d'inscription à ce même niveau d'autre part. La présente étude veut tout à la fois tracer les tendances historiques en regard de l'utilisation des diplômés d'un doctorat des universités canadiennes et jeter un regard sur les perspectives d'avenir.

Traditionnellement, environ $65 \%$ des diplômés d'un doctorat s'orientaient vers des carrières dites pédagogiques. A l'heure actuelle, à cause du jeune âge du personnel enseignant, on y compte peu de retraités ou de gens qui décèdent, ce qui explique des demandes annuelles de seulement 500 nouveaux diplômés d'un doctorat pour remplir les postes existants. Toutefois, les universités canadiennes décernent actuellement environ 2,000 doctorats par an (y compris les canadiens qui reviennent d'un stage dans un autre pays).

D'ailleurs, il est à prévoir que ce déséquilibre aura tendance à se perpétuer. En se basant sur les 13,000 inscriptions actuelles à des programmes de doctorat, une projection a été faite de l'offre des cardidats en regard des années 1977-78 à 1981-82, pour 45 disciplines. En juxtaposant ces projections de l'offre par rapport à la demande probable pour des enseignants universitaires, un surplus se révèle dans presque toutes les disciplines. Un cycle de pénurie et de surplus paraît s'être développé dans certaines disciplines. Ces simulations proviennent des hypothèses contenues dans deux appendices et vingt-six tableaux ciannexés.

De plus, cette étude se penche également sur d'autres aspects de la situation des doctorats au Canada: une histoire de la croissance de l'enseignement supérieur; des écarts dans le rapport des inscriptions au doctorat dans de différentes disciplines; les programmes de soutien pour des candidats au doctorat et l'immigration d'enseignants universitaires.

Ces information fournissent un aperçu général des dimensions multiples de l'utilité du doctorat au Canada.

\section{INTRODUCTION}

By 1971 , the imbalance between the growing supply of $\mathrm{Ph} . \mathrm{D}$. graduates and the declining demand for them, particularly in the university sector, had become apparent. The Economic Council explored this issue in a report published in Canadian Higher Education in the Seventies in 1972. 'The information available then was limited, but now many of the questions raised can be answered more authoritatively on the basis of recent data. The purpose of this report is to provide that data, and at the same time, discuss some of the issues.

This report is organized into four sections and two appendices.

The first section presents a statistical outline of the Ph.D. situation in Canada: the Ph.D. population, (e.g. employment sector, occupation, immigration status and university teachers' characteristics), degrees granted, and employment trends.

The second investigates the enrolment pattern of full-time and part-time doctoral students by field of study and legal residence status. It also gives, on a provincial basis, the number of Canada Student Loan Plan recipients, and the number of Canada Council Doctoral Fellows, by discipline.

1 "The Ph.D. Dilemma in Canada: A Case Study". This study also provided a selected bibliography on the subject in the Canadian context, p. 128-131. 
The third section deals with the structure of doctoral programs at Canadian universities, particularly the increasing number of graduate programs, and discusses the growth pattern for selected disciplines. In addition, Ph.D. enrolment is related to the number of degrees granted by discipline.

The last section focuses on the anticipated supply and demand of Ph.Ds in the university sector from 1977-78 to 1981-82.

\section{THE PH.D. SITUATION: BASIC STATISTICS}

\section{Employment}

The Highly Qualified Manpower Survey of 1973 presented, for the first time, an excellent overview of how Canada's Ph.D. population was employed. ${ }^{2}$ By 1973 , according to this survey, 27,410 residents of Canada had earned a doctorate. Of those who were part of the labour force, $64.8 \%$ were working in education (Table 1). The various levels of government employed $14.7 \%$ (11.7\% in the federal government alone), and the industrial sector accounted for about $13.5 \%$.

An occupational breakdown reflects this distribution of Ph.Ds among employment sectors. About half ( $50.8 \%$ ) were university teachers, while other educational institutions employed $4.4 \%$ (Table 2 ). In addition, $4.7 \%$ were educational administrators. Chemists, geologists, engineers, and similar scientific occupations constituted a large component (20.9\%). Another group (7.8\%) functioned as administrators and managers in both government and industry.

\section{Replacement}

A unique characteristic of Canada's Ph.D. population is its relative youth. In the educational sector, two-thirds are younger than 44 , and their average age has been estimated at 40 . This means that for the next ten to fifteen years attrition due to retirement and death will be low. The current annual attrition rate, about $1.3 \%$, opens about 500 replacement positions for Ph.Ds in all sectors of employment each year. However, around 2,000 Ph.Ds become available for employment annually, and demand in education and government is not expanding. The imbalance is apparent.

But the present age structure suggests a substantial replacement demand for Ph.Ds in 15 years, particularly in education. Since the average time to complete a Ph.D. is five years from the masters or equivalent level, the question of supply needs to be explored before the late eighties.

\section{Citizenship}

In the past, Canada has relied heavily on immigrants for highly qualified manpower: $57.6 \%$ of the $1973 \mathrm{Ph} . \mathrm{D}$. population were immigrants. In addition, a large number of Canadians have obtained their degrees abroad. The 1973 survey showed that $31.9 \%$ of the Ph.D. population, including Canadian citizens and landed immigrants, completed their doctoral studies in the United States, and $22.9 \%$ in Europe (Table 3).

${ }^{2}$ Highlights of this information have been discussed in a separate article "Profile of Ph.Ds in Canada", Canadian Statistical Review (July 1976). 
TABLE 1

EMPLOYMENT OF PH.DS BY INDUSTRIAL

SECTOR AND BY AGE, 1973

\begin{tabular}{|c|c|c|c|c|c|}
\hline INDUSTRIAL SECTOR & $\begin{array}{c}\text { YOUNGER THAN } \\
34 \\
\%\end{array}$ & $34-44$ & $\begin{array}{c}\text { OLDER THAN } \\
44 \\
\%\end{array}$ & NUMBER & PERCENT* \\
\hline $\begin{array}{l}\text { Primary industries } \\
\text { (e.g., agriculture, mining) }\end{array}$ & 19.6 & 37.1 & 43.3 & 485 & $(1.8)$ \\
\hline Manufacturing & 26.7 & 36.4 & 36.4 & 1,290 & $(4.9)$ \\
\hline $\begin{array}{l}\text { Service industries } \\
\text { (e.g., transportation, trade, } \\
\text { finance) }\end{array}$ & 24.7 & 27.2 & 46.9 & 395 & $(1.5)$ \\
\hline Education and related & 24.1 & 43.0 & 33.0 & 17,120 & $(64.8)$ \\
\hline Health and Welfare Services & 21.5 & 39.0 & 39.5 & 1,000 & $(3.8)$ \\
\hline Religious Organizations & 1.6 & 18.5 & 79.8 & 620 & $(2.3)$ \\
\hline $\begin{array}{l}\text { Other Services } \\
\text { (e.g, community, business, } \\
\text { persona1) }\end{array}$ & 18.2 & 20.5 & 56.8 & 225 & $(0.8)$ \\
\hline Business Management & 26.0 & 35.4 & 39.0 & 1,290 & $(4.9)$ \\
\hline Federal Administration & 20.5 & 37.3 & 42.0 & 3,090 & $(11.7)$ \\
\hline Provincial Administration & 24.5 & 34.7 & 42.2 & 735 & $(2.8)$ \\
\hline Municipal Administration & 30.0 & 40.0 & 40.0 & 50 & $(0.2)$ \\
\hline $\begin{array}{l}\text { Industry as unspecified or } \\
\text { undefined }\end{array}$ & 41.7 & 29.2 & 29.2 & 120 & $(0.4)$ \\
\hline TOTAL & 23.1 & 39.1 & 37.8 & 26,405 & $(100.0)$ \\
\hline
\end{tabular}

* Percentage in brackets provide breakdown by industrial sector. Source: Statistics Canada, unpublished data.

Examination of the country of birth of foreign-born $\mathrm{Ph}$.Ds reveals that $25.0 \%$ came from the United Kingdom, 24.5\% from the United States and a similar proportion from other European countries combined (Table 4). Almost $45 \%$ of them entered Canada between 1966 and June 1971. ${ }^{3}$

\section{University Teachers}

Historically, more than half of the Ph.Ds have been employed as university teachers. During the last 20 years, Canadian universities underwent remarkable growth. The number

3 The mailing list for the Highly Qualified Manpower Survey of 1973 was derived from the 1971 Census. Consequently, no one with a Ph.D. who immigrated to Canada between June 1971 and fall of 1973 was included. 


\begin{tabular}{|c|c|c|c|c|c|c|}
\hline $\begin{array}{l}\text { SELECTED } \\
\text { OCCUPATION }\end{array}$ & MALE & $\begin{array}{l}\text { PER- } \\
\text { CENT }\end{array}$ & FEMALE & $\begin{array}{l}\text { PER- } \\
\text { CENT }\end{array}$ & TOTAL & $\begin{array}{l}\text { PER- } \\
\text { CENT'大 }\end{array}$ \\
\hline Government Administrators & 455 & 94.8 & 20 & 5.2 & 480 & $(1.8)$ \\
\hline General Managers and Senior Officers & 580 & 100.0 & -- & 0.0 & 580 & $(2.2)$ \\
\hline Administrators in Teaching & 1,175 & 94.4 & 65 & 5.6 & 1,245 & $(4.7)$ \\
\hline Other Managers and Administrators & 9.15 & 94.3 & 55 & 5.7 & 970 & $(3.8)$ \\
\hline Chemists & 1,490 & 94.6 & 90 & 5.4 & 1,575 & $(6.0)$ \\
\hline Geologists & 605 & 99.2 & 10 & 0.8 & 610 & $(2.3)$ \\
\hline Agriculturists and Related & 570 & 100.0 & -- & 0.0 & 570 & $(2.1)$ \\
\hline Other Natural Scientists & 1,150 & 94.3 & 75 & 5.7 & 1,220 & $(4.6)$ \\
\hline Engineers, Architects, System Analysts & 1,490 & 96.1 & 60 & 3.9 & 1,550 & $(5.9)$ \\
\hline Economists & 185 & 94.9 & 5 & 5.1 & 195 & $(0.7)$ \\
\hline Psychologists & 295 & 80.8 & 65 & 19.2 & 365 & $(1.4)$ \\
\hline Judges and Lawyers & 115 & 92.0 & 10 & 8.0 & 125 & $(0.5)$ \\
\hline Other Social Scientists & 200 & 78.4 & 60 & 21.6 & 255 & $(1.0)$ \\
\hline Ministers of Religion & 570 & 96.6 & 20 & 0.0 & 590 & $(2.2)$ \\
\hline University Teachers & 12,155 & 90.5 & 1,270 & 9.5 & 13,425 & $(50.8)$ \\
\hline Elementary and Secondary Teachers & 260 & 82.5 & 55 & 17.5 & 315 & $(1.2)$ \\
\hline Post-Secondary Non-University Teachers & 410 & 82.0 & 90 & 18.0 & 500 & $(1.9)$ \\
\hline Other Teachers and Related & 225 & 67.2 & 110 & 32.8 & 335 & $(1.3)$ \\
\hline Physicians and Surgeons & 320 & 95.5 & 10 & 4.5 & 335 & $(1.3)$ \\
\hline Dentists & 20 & 100.0 & -- & 0.0 & 25 & $(0.1)$ \\
\hline Pharmacists & 50 & 76.9 & 10 & 23.1 & 65 & $(0.2)$ \\
\hline Other Health Occupations & 50 & 90.9 & 5 & 9.1 & 55 & $(0.2)$ \\
\hline Writers, Editors and Related Occupations & 140 & 77.8 & 35 & 22.2 & 180 & $(0.7)$ \\
\hline Clerical, and Service & 105 & 95.5 & 10 & 4.5 & 110 & $(0.4)$ \\
\hline Military and Policy Officers & 160 & 97.0 & 5 & 3.0 & 165 & $(0.6)$ \\
\hline Other Occupations & 205 & 97.6 & 10 & 2.4 & 210 & $(0.8)$ \\
\hline Not Stated & 105 & 84.0 & 20 & 16.0 & 125 & $(0.5)$ \\
\hline TOTAL & 23,985 & & 2,165 & 9.0 & 26,405 & $(100.0)$ \\
\hline
\end{tabular}

* Percentage in brackets provide breakdown by occupation.

Source: Statistics Canada, unpublished data.

of full-time teachers increased sixfold, from less than 5,000 in 1956-57 to almost 30,000 in 1974-75 (Table 5). The most spectacular expansion took place in the social sciences, which grew from 931 to 9,863 . In comparison, the physical and applied sciences increased from 1,491 to 6,637 .

Between 1963-64 and 1972-73 the average annual increase in the number of university teachers was about 2,000 , excluding the few hundred replacement positions that were filled. This meant that between 2,200 and 2,400 full-time teachers were hired each year, and approximately half had a Ph.D. Many of them were landed immigrants. According to immigration statistics, 17,713 immigrants whose intended occupation was university teaching were admitted to Canada between 1962 and $1974 .^{4}$ Most came from the United

${ }^{4}$ This refers to intention of immigrants, not positions obtained. There is another group of immigrants whose original intended occupation was not university teaching, but who were eventually employed by universities. 
GEOGRAPHIC ORIGIN OF PH.DS, 1973

\begin{tabular}{|c|c|c|c|}
\hline & $\begin{array}{c}\text { NUMBERS } \\
\text { BY } \\
\text { COUNTRY }\end{array}$ & $\begin{array}{c}\text { NUMBERS } \\
\text { BY } \\
\text { REGION }\end{array}$ & PERCENT \\
\hline \multicolumn{4}{|l|}{ Canada } \\
\hline $\begin{array}{l}\text { Atlantic Provinces } \\
\text { Quebec } \\
\text { Ontario } \\
\text { Manitoba } \\
\text { Saskatchewan } \\
\text { Alberta } \\
\text { British Columbia }\end{array}$ & 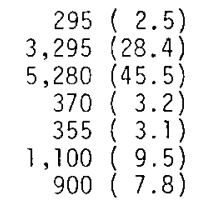 & & \\
\hline TOTAL, CANADA & $(100.0)$ & 11,595 & 42.4 \\
\hline United States & & 8,730 & 31.9 \\
\hline \multicolumn{4}{|l|}{ Europe } \\
\hline $\begin{array}{l}\text { Czechoslovakia } \\
\text { France } \\
\text { Germany } \\
\text { Italy \& Holy See } \\
\text { Switzerland } \\
\text { United Kingdom } \\
\text { Others }\end{array}$ & 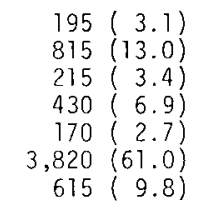 & & \\
\hline TOTAL, EUROPE & & 6,260 & 22.9 \\
\hline Australia \& New Zealand & & 260 & 0.9 \\
\hline Asia (primarily India) & & 370 & 1.4 \\
\hline $\begin{array}{r}\text { other countries } \\
\text { (e.g., Africa, } \\
\text { Latin America) }\end{array}$ & & 160 & 0.6 \\
\hline TOTAL, ALL COUNTRIES & & 27,410 & 100.0 \\
\hline
\end{tabular}

Percentage in brackets provides regional breakdowns.

States (46.3\%) and Great Britain (19.3\%) (Table 6). Between 1972 and 1974, more than 1,200 immigrants whose intended occupation was university teaching entered the country each year, although the number of available positions had drastically declined. Unfortunately, information about the Ph.D. qualifications of landed immigrants who plan to teach at a university is not available. Table 7 shows characteristics such as average age and salary, proportion of females, and citizenship of university teachers in 1973-74. Faculties have been grouped into 47 disciplines under eight teaching fields. As an illustration, there were 1,465 (5.1\%) faculty members teaching English; 1,229 (4.3\%) 
TABLE 4

FOREIGN BORN PH.DS BY COUNTRY OF BIRTH AND PERIOD OF IMMIGRATION, 1973

\begin{tabular}{|c|c|c|c|c|c|c|c|c|c|c|}
\hline & $\begin{array}{c}\text { BEFORE } \\
\text { No. }\end{array}$ & $\begin{array}{c}1955 \\
\%\end{array}$ & No. & $6-60$ & NO. & $1-65$ & $\begin{array}{l}966-\mathrm{JU} \\
\text { No. }\end{array}$ & E 1971 & $\begin{array}{l}\text { TOTAL } \\
\text { NUMBER }\end{array}$ & PERCENT* \\
\hline \multicolumn{11}{|l|}{ EUROPE } \\
\hline United Kingdom & 1,040 & 29.9 & 595 & 37.1 & 555 & 15.0 & 1,285 & 36.9 & 3,480 & $(25.0)$ \\
\hline Germany & 225 & 46.4 & 60 & 12.4 & 70 & 14.4 & 135 & 27.8 & 485 & $(3.5)$ \\
\hline Soviet Union & 335 & 77.9 & 30 & 7.0 & 15 & 3.5 & 50 & 11.6 & 430 & $(3.1)$ \\
\hline Poland & 225 & 63.4 & 40 & 11.3 & 20 & 5.6 & 70 & 19.7 & 355 & $(2.6)$ \\
\hline France & 85 & 27.0 & 20 & 6.3 & 75 & 23.8 & 125 & 39.7 & 315 & $(2.9)$ \\
\hline Netherlands & 145 & 56.9 & 60 & 23.5 & 45. & 17.6 & 15 & 5.9 & 255 & $(1.8)$ \\
\hline Hungary & 85 & 29.8 & 130 & 45.6 & 20 & 7.0 & 55 & 19.3 & 285 & $(2.0)$ \\
\hline Czechos lavakia & 70 & 21.5 & 5 & 1.5 & 10 & 3.1 & 225 & 69.2 & 325 & $(2.3)$ \\
\hline Yugoslavia & 60 & 57.1 & 5 & 4.8 & 15 & 14.3 & 20 & 19.0 & 105 & $(0.8)$ \\
\hline Austria & 70 & 66.7 & 10 & 9.5 & 10 & 9.5 & 15 & 14.3 & 105 & $(0.8)$ \\
\hline Belgium & 50 & 34.5 & 15 & 10.3 & 45 & 31.0 & 35 & 24.1 & 145 & (1.0) \\
\hline Greece & 25 & 25.0 & 45 & 45.0 & -- & 0.0 & 25 & 25.0 & 100 & $(0.7)$ \\
\hline Spain \& Portugal & 10 & 10.0 & 15 & 15.0 & 25 & 25.0 & 50 & 50.0 & 100 & $(0.7)$ \\
\hline Others: Europe & 145 & 33.0 & 55 & 12.5 & 75 & 17.0 & 155 & 35.2 & 440 & $(3.2)$ \\
\hline United States & 435 & 12.7 & 220 & 6.4 & 600 & 17.6 & 2,110 & 61.8 & 3,415 & $(24.5)$ \\
\hline India & 60 & 5.7 & 95 & 9.0 & 250 & 23.8 & 665 & 62.4 & 1,050 & $(7.5)$ \\
\hline China & 80 & 16.7 & 50 & 10.4 & 125 & 26.0 & 215 & 44.8 & 480 & $(3.4)$ \\
\hline Japan & 25 & 18.5 & -- & 0.0 & 25 & 18.5 & 80 & 59.3 & 135 & $(1.0)$ \\
\hline Egypt \& Libya & 10 & 4.5 & 50 & 22.7 & 60 & 27.3 & 105 & 47.7 & 220 & $(1.6)$ \\
\hline other countries & 60 & 10.3 & 75 & 12.9 & 140 & 24.1 & 295 & 50.9 & 580 & $(4.2)$ \\
\hline TOTAL, ALL COUNTRIES & 3,475 & 25.0 & 1,780 & 12.8 & 2,430 & 17.5 & 6,230 & 44.8 & 13,915 & \\
\hline
\end{tabular}

*Percent in brackets show geographic distribution

psychology and $1,162(3.5 \%)$ chemistry. Two-thirds were Canadian citizens, with some variations among disciplines; $13 \%$ were female, but women were concentrated in fine arts, modern languages, literature, education, social work, and household sciences.

The proportion who held a doctorate was $56.8 \%$ for all disciplines, with a high of $82.0 \%$ in the physical sciences.

\section{Federal Government Employees}

Traditionally, the federal government has also been a major employer of doctoral graduates. According to the Highly Qualified Manpower Survey, 3,090 Ph.Ds (11\%) worked for the government in 1973. From Public Service Commission data it was possible to ascertain the length of employment and the discipline of study of the 2,293 hired under the Public Service Employment Act (Table 8). ${ }^{5}$

${ }^{5}$ Since employees of a number of federal agencies such as the National Research Council, the Economic Council and crown corporations were not part of the "Data Stream" of the Commission, this figure underestimates the actual number of $\mathrm{Ph} . \mathrm{Ds}$ in the public service. Moreover, some Ph.D.- holders in the government sector might not have identified themselves as such. 
Table 5
Full Time University Teachers by Fleld of Study, 1956-57 to 1974-75

\begin{tabular}{|c|c|c|c|c|c|c|c|c|c|c|c|c|c|c|}
\hline & $\begin{array}{c}\text { Soc1al } \\
\text { Sctences }\end{array}$ & $\begin{array}{c}\text { Increase } \\
\text { over } \\
\text { previouq } \\
\text { year }\end{array}$ & Humenteles & $\begin{array}{c}\text { Increase } \\
\text { over } \\
\text { previous } \\
\text { year }\end{array}$ & $\begin{array}{l}\text { Sub-total } \\
\text { human } \\
\text { ociences }\end{array}$ & $\begin{array}{c}\text { Increase } \\
\text { over } \\
\text { prevfous } \\
\text { year }\end{array}$ & Life Sciences & $\begin{array}{c}\text { Increase } \\
\text { over } \\
\text { prevfous } \\
\text { year }\end{array}$ & $\begin{array}{l}\text { Physical } \\
\text { and } \\
\text { Applied } \\
\text { Sclences }\end{array}$ & $\begin{array}{c}\text { Increase } \\
\text { over } \\
\text { previous } \\
\text { year }\end{array}$ & $\begin{array}{c}\text { Sub. } \\
\text { total } \\
\text { Natural } \\
\text { Sciences }\end{array}$ & $\begin{array}{c}\text { Increase } \\
\text { over } \\
\text { previous } \\
\text { year }\end{array}$ & $\begin{array}{l}\text { Grand } \\
\text { Total }\end{array}$ & $\begin{array}{c}\text { Increase } \\
\text { over } \\
\text { previous } \\
\text { year }\end{array}$ \\
\hline 1956.1957 & 931 & 97 & 1,181 & 99 & 2,112 & 196 & 1,370 & -95 & 1,491 & 74 & 2,861 & -21 & 4,973 & 175 \\
\hline $1957.1958 *$ & 1,028 & 98 & 1,280 & 100 & 2,308 & 198 & 1,275 & -94 & 1,565 & 73 & 2,840 & -21 & 5,148 & 177 \\
\hline 1958-1959 & 1,126 & 150 & 1,380 & 144 & 2,506 & 294 & 1,181 & 67 & 1,638 & 202 & 2,819 & 269 & 5,325 & 563 \\
\hline $\begin{array}{l}1959-1960 * \\
1960-1961\end{array}$ & 1,276 & 151 & 1,524 & 145 & 2,800 & 296 & 1,248 & 69 & 1,840 & 201 & 3,088 & 270 & 5,888 & 566 \\
\hline $\begin{array}{l}1960-1961 \\
1961-1962 *\end{array}$ & $\begin{array}{l}1,427 \\
1,630\end{array}$ & 203 & $\begin{array}{l}1,669 \\
1,878\end{array}$ & 209 & $\begin{array}{l}3,096 \\
3,508\end{array}$ & 412 & $\begin{array}{l}1,317 \\
1,432\end{array}$ & 115 & $\begin{array}{l}2,041 \\
2,232\end{array}$ & 191 & $\begin{array}{l}3,358 \\
3,664\end{array}$ & 306 & $\begin{array}{l}6,454 \\
7,172\end{array}$ & 718 \\
\hline $1962-1963$ & 1,834 & $\begin{array}{l}204 \\
376\end{array}$ & 2,087 & $\begin{array}{l}209 \\
397\end{array}$ & 3,921 & $\begin{array}{l}413 \\
773\end{array}$ & 1,546 & $\begin{array}{l}114 \\
194\end{array}$ & 2,423 & $\begin{array}{l}191 \\
268\end{array}$ & 3,969 & $\begin{array}{l}305 \\
462\end{array}$ & 7,890 & $\begin{array}{r}718 \\
1,235\end{array}$ \\
\hline $\begin{array}{l}1963-1964 \\
1954-1965 *\end{array}$ & $\begin{array}{l}2,210 \\
2,671\end{array}$ & 461 & $\begin{array}{l}2,484 \\
2,945\end{array}$ & 461 & $\begin{array}{l}4,694 \\
5,616\end{array}$ & 922 & $\begin{array}{l}1,740 \\
1,960\end{array}$ & 220 & $\begin{array}{l}2,691 \\
3,027\end{array}$ & 336 & $\begin{array}{l}4,431 \\
4,987\end{array}$ & 556 & $\begin{array}{r}9,125 \\
10,603\end{array}$ & 1,478 \\
\hline $1965-1966$ & 3,133 & $\begin{array}{l}462 \\
771\end{array}$ & 3,406 & $\begin{array}{l}461 \\
588\end{array}$ & 6,539 & $\begin{array}{r}923 \\
1,359\end{array}$ & $\begin{array}{l}1,960 \\
2,183\end{array}$ & $\begin{array}{l}223 \\
468\end{array}$ & 3,363 & $\begin{array}{l}336 \\
480\end{array}$ & $\begin{array}{l}4,987 \\
5,546\end{array}$ & $\begin{array}{l}559 \\
948\end{array}$ & $\begin{array}{l}10,603 \\
12,085\end{array}$ & $\begin{array}{l}1,482 \\
2,307\end{array}$ \\
\hline 1966-1967 k & 3.904 & 772 & $\begin{array}{l}3,994 \\
4,583\end{array}$ & 589 & $\begin{array}{l}7,898 \\
9,259\end{array}$ & 1,361 & 2,651 & 470 & 3,843 & 480 & 6,494 & 950 & 14,392 & 2,311 \\
\hline $\begin{array}{l}1967-1968 \\
1968-1969\end{array}$ & $\begin{array}{l}4,676 \\
5,424\end{array}$ & 748 & $\begin{array}{l}4,583 \\
5,073\end{array}$ & 490 & $\begin{array}{r}9,259 \\
10,497\end{array}$ & 1,238 & $\begin{array}{l}3,121 \\
3,596\end{array}$ & 475 & $\begin{array}{l}4,323 \\
4,771\end{array}$ & 448 & $\begin{array}{l}7,444 \\
8,367\end{array}$ & 923 & $\begin{array}{l}16,703 \\
18,864\end{array}$ & 2,161 \\
\hline $1909-1970$ & 6,430 & $\begin{array}{l}1,006 \\
1,098\end{array}$ & 5,850 & $\begin{array}{l}777 \\
776\end{array}$ & 12,280 & $\begin{array}{l}1,783 \\
1,874\end{array}$ & 4,087 & $\begin{array}{l}491 \\
702\end{array}$ & 5,472 & $\begin{array}{l}701 \\
189\end{array}$ & 9,559 & $\begin{array}{r}1,192 \\
891\end{array}$ & 21,839 & $\begin{array}{l}2,975 \\
2,705\end{array}$ \\
\hline $\begin{array}{l}1970-1971 \\
1971-1972\end{array}$ & $\begin{array}{l}7,528 \\
8,598\end{array}$ & 1,070 & $\begin{array}{l}6,626 \\
6,972\end{array}$ & 346 & $\begin{array}{l}14,154 \\
15,570\end{array}$ & 1,416 & $\begin{array}{l}4,789 \\
5,244\end{array}$ & 455 & $\begin{array}{l}5,661 \\
6,149\end{array}$ & 488 & $\begin{array}{l}10,450 \\
11,393\end{array}$ & 943 & $\begin{array}{l}24,604 \\
26,963\end{array}$ & 2.359 \\
\hline $1972-1973$ & 8,846 & $\begin{array}{l}248 \\
411\end{array}$ & 7,138 & $\begin{array}{r}166 \\
-90\end{array}$ & 15,984 & $\begin{array}{l}414 \\
321\end{array}$ & 5,493 & $\begin{array}{l}249 \\
341\end{array}$ & 6,393 & $\begin{array}{r}244 \\
7\end{array}$ & 11,886 & $\begin{array}{l}493 \\
348\end{array}$ & 27,870 & $\begin{array}{l}907 \\
669\end{array}$ \\
\hline $\begin{array}{l}1973-1974 \\
1974-1975 \text { \# }\end{array}$ & $\begin{array}{l}9,257 \\
9,863\end{array}$ & 606 & $\begin{array}{l}7,048 \\
7,168\end{array}$ & 120 & $\begin{array}{l}16,305 \\
17,031\end{array}$ & 726 & $\begin{array}{l}5,834 \\
6,042\end{array}$ & 208 & $\begin{array}{l}6,400 \\
6,637\end{array}$ & 237 & $\begin{array}{l}12,234 \\
12,679\end{array}$ & 445 & $\begin{array}{l}28,539 \\
29,710\end{array}$ & 1,171 \\
\hline
\end{tabular}

** Includes for the first time Ryerson Polytechnical Institure with 623 faculty members, accounting for over $50 \%$ of the increase.

Source: Statistica Canada, unpublished data. 
Immigrants to Canada by Country of Last Permanent Residence and Intended Occupation: "University Teaching", 1962 to 1974

\begin{tabular}{|c|c|c|c|c|c|c|c|c|c|c|c|c|c|c|}
\hline & 1962 & 1963 & 1964 & 1965 & 1966 & 1967 & 1968 & 1969 & 1970 & 1971 & 1972 & 1973 & 1974 & $\begin{array}{l}\text { Total } \\
1962 \text { to } 1974\end{array}$ \\
\hline $\begin{array}{l}\text { Great Britain } \\
\text { Per Cent }\end{array}$ & $\begin{array}{r}95 \\
24.4\end{array}$ & $\begin{array}{r}160 \\
29.7\end{array}$ & $\begin{array}{r}195 \\
29.0\end{array}$ & $\begin{array}{r}271 \\
25.0\end{array}$ & $\begin{array}{r}353 \\
25.0\end{array}$ & $\begin{array}{r}457 \\
23.6\end{array}$ & $\begin{array}{r}545 \\
23.9\end{array}$ & $\begin{array}{r}499 \\
20.8\end{array}$ & $\begin{array}{r}284 \\
15.1\end{array}$ & $\begin{array}{r}143 \\
10.5\end{array}$ & $\begin{array}{r}117 \\
11.3\end{array}$ & $\begin{array}{r}155 \\
10.5\end{array}$ & $\begin{array}{r}140 \\
11.7\end{array}$ & $\begin{array}{r}3.414 \\
19.3\end{array}$ \\
\hline $\begin{array}{l}\text { France } \\
\quad \text { Per Cent }\end{array}$ & $\begin{array}{r}25 \\
6.4\end{array}$ & $\begin{array}{r}30 \\
5.6\end{array}$ & $\begin{array}{r}27 \\
4.0\end{array}$ & $\begin{array}{r}42 \\
3.9\end{array}$ & $\begin{array}{r}69 \\
4.9\end{array}$ & $\begin{array}{r}81 \\
4.1\end{array}$ & $\begin{array}{r}87 \\
3.8\end{array}$ & $\begin{array}{r}90 \\
3.8\end{array}$ & $\begin{array}{l}105 \\
5.5\end{array}$ & $\begin{array}{r}77 \\
5.7\end{array}$ & $\begin{array}{r}73 \\
7.2\end{array}$ & $\begin{array}{r}94 \\
6.3\end{array}$ & $\begin{array}{r}86 \\
7.2\end{array}$ & $\begin{array}{l}886 \\
5.0\end{array}$ \\
\hline $\begin{array}{l}\text { Other Western European Countries } \\
\text { Per Cent }\end{array}$ & $\begin{array}{r}41 \\
10.5\end{array}$ & $\begin{array}{r}39 \\
7.2\end{array}$ & $\begin{array}{r}58 \\
8.6\end{array}$ & $\begin{array}{r}77 \\
7.1\end{array}$ & $\begin{array}{r}97 \\
6.9\end{array}$ & $\begin{array}{l}142 \\
7.2\end{array}$ & $\begin{array}{l}175 \\
7.7\end{array}$ & $\begin{array}{l}157 \\
6.5\end{array}$ & $\begin{array}{l}155 \\
8.2\end{array}$ & $\begin{array}{r}61 \\
4.5\end{array}$ & $\begin{array}{r}75 \\
7.3\end{array}$ & $\begin{array}{r}96 \\
6.5\end{array}$ & $\begin{array}{r}92 \\
7.7\end{array}$ & $\begin{array}{r}1,265 \\
7.1\end{array}$ \\
\hline $\begin{array}{l}\text { India, Pakistan } \\
\text { Per Cent }\end{array}$ & $\begin{array}{r}14 \\
3.6\end{array}$ & $\begin{array}{r}38 \\
7.1\end{array}$ & $\begin{array}{r}31 \\
4.6\end{array}$ & $\begin{array}{r}81 \\
7.5\end{array}$ & $\begin{array}{r}86 \\
6.1\end{array}$ & $\begin{array}{l}131 \\
6.6\end{array}$ & $\begin{array}{r}99 \\
4.3\end{array}$ & $\begin{array}{l}177 \\
7.4\end{array}$ & $\begin{array}{l}120 \\
6.4\end{array}$ & $\begin{array}{r}87 \\
6.4\end{array}$ & $\begin{array}{r}54 \\
5.2\end{array}$ & $\begin{array}{r}89 \\
6.0\end{array}$ & $\begin{array}{r}56 \\
4.7\end{array}$ & $\begin{array}{r}1,063 \\
6.0\end{array}$ \\
\hline $\begin{array}{r}\text { Other Asta } \\
\text { Per Cent }\end{array}$ & $\begin{array}{r}4 \\
1.0\end{array}$ & $\begin{array}{r}14 \\
2.6\end{array}$ & $\begin{array}{r}6 \\
0.9\end{array}$ & $\begin{array}{r}27 \\
2.5\end{array}$ & $\begin{array}{r}49 \\
3.5\end{array}$ & $\begin{array}{r}89 \\
4.5\end{array}$ & $\begin{array}{l}124 \\
5.4\end{array}$ & $\begin{array}{l}128 \\
5.3\end{array}$ & $\begin{array}{l}166 \\
8.8\end{array}$ & $\begin{array}{r}80 \\
5.9\end{array}$ & $\begin{array}{r}53 \\
5.1\end{array}$ & $\begin{array}{r}180 \\
12.2\end{array}$ & $\begin{array}{l}113 \\
9.4\end{array}$ & $\begin{array}{r}1,033 \\
5.8\end{array}$ \\
\hline $\begin{array}{l}\text { United States } \\
\text { Per Cent }\end{array}$ & $\begin{array}{r}174 \\
44.6\end{array}$ & $\begin{array}{r}208 \\
38.6\end{array}$ & $\begin{array}{r}267 \\
39.7\end{array}$ & $\begin{array}{r}477 \\
44.0\end{array}$ & $\begin{array}{r}615 \\
43.6\end{array}$ & $\begin{array}{r}857 \\
43.2\end{array}$ & $\begin{array}{r}1,013 \\
44.4\end{array}$ & $\begin{array}{r}1,040 \\
43.4\end{array}$ & $\begin{array}{r}918 \\
48.7\end{array}$ & $\begin{array}{r}774 \\
57.0\end{array}$ & $\begin{array}{r}556 \\
53.9\end{array}$ & $\begin{array}{r}708 \\
47.8\end{array}$ & $\begin{array}{r}588 \\
49.1\end{array}$ & $\begin{array}{r}8,195 \\
46.3\end{array}$ \\
\hline $\begin{array}{l}\text { All other countries } \\
\text { Per Cent }\end{array}$ & $\begin{array}{r}37 \\
9.5\end{array}$ & $\begin{array}{r}50 \\
9.3\end{array}$ & $\begin{array}{r}88 \\
13.1\end{array}$ & $\begin{array}{r}109 \\
10.1\end{array}$ & $\begin{array}{r}141 \\
10.0\end{array}$ & $\begin{array}{r}229 \\
13.5\end{array}$ & $\begin{array}{r}237 \\
10.4\end{array}$ & $\begin{array}{r}307 \\
12.8\end{array}$ & $\begin{array}{l}138 \\
7.3\end{array}$ & $\begin{array}{r}136 \\
10.0\end{array}$ & $\begin{array}{r}103 \\
10.0\end{array}$ & $\begin{array}{r}159 \\
10.7\end{array}$ & $\begin{array}{r}123 \\
10.2\end{array}$ & $\begin{array}{r}1,857 \\
10.5\end{array}$ \\
\hline TOTAL & 390 & 539 & 672 & 1,084 & 1.410 & 1,986 & 2,280 & 2,398 & 1,886 & 1,358 & 1,031 & 1,481 & 1,198 & 17,713 \\
\hline
\end{tabular}

Source: Department of Manpower and Immigration, unpublished Data. 
Table 7

Characteristics of University Teachers by Disclpline, 1973-74

\begin{tabular}{|c|c|c|c|c|c|c|c|}
\hline Discipline & Number & Percent & $\begin{array}{l}\text { Percentage } \\
\text { with } \\
\text { Doctorate }\end{array}$ & $\begin{array}{c}\text { Average } \\
\text { Age }\end{array}$ & $\begin{array}{l}\text { Average } \\
\text { Salary }\end{array}$ & $\begin{array}{l}\text { Percentage } \\
\text { Canadian } \\
\text { Citizen }\end{array}$ & $\begin{array}{l}\text { Percentage } \\
\text { Female }\end{array}$ \\
\hline & & & & & $\$$ & & \\
\hline $\begin{array}{l}\text { Physical Education } \\
\text { Education }\end{array}$ & $\begin{array}{r}632 \\
2,050\end{array}$ & $\begin{array}{l}2.2 \\
7.2\end{array}$ & $\begin{array}{l}29.3 \\
43.5\end{array}$ & - & $\begin{array}{l}15,659 \\
18,255\end{array}$ & - & - \\
\hline Education & 2,050 & 7.2 & 43.5 & - & & - & - \\
\hline Sub-total Education & 2,682 & 9.4 & 40.1 & 40.6 & 17,632 & 76.3 & 21.3 \\
\hline Music & 425 & 1.5 & 22.8 & - & 15,670 & - & - \\
\hline Flne \& Applied Arts & 623 & 2.2 & 15.9 & - & 15.408 & - & - \\
\hline Sub-total fine Arts & 1,048 & 3.7 & 18.7 & 39.9 & 15,513 & 59.6 & 18.7 \\
\hline Classics & 269 & 1.0 & 62.0 & 41.5 & 17,826 & - & 14.9 \\
\hline History & 1,037 & 3.6 & 68.0 & 39.4 & 17,320 & 66.2 & 7.8 \\
\hline Library and Records Sclence & 93 & 0.3 & 23.9 & 44.8 & 18,314 & - & 50.0 \\
\hline Mass Media Studies & 83 & 0.3 & 17.7 & 40.7 & 16,829 & - & 5.0 \\
\hline English & 1,465 & 5.1 & 60.7 & 40.6 & 16,814 & 59.4 & 20.2 \\
\hline French & 776 & 2.7 & 47.8 & 40.4 & 16.099 & 63.1 & 28.6 \\
\hline German & 224 & 0.8 & 72.3 & 41.3 & 16,597 & - & 23.0 \\
\hline Spanish & 156 & 0.6 & 52.3 & 40.6 & 15,738 & - & 28.1 \\
\hline Other Modern Languages & 667 & 2.3 & 49.9 & 40.2 & 16,581 & - & 19.9 \\
\hline Philosophy & 693 & 2.5 & 67.0 & 39.9 & 17,934 & 59.4 & 5.4 \\
\hline Religious Studies & 537 & 1.9 & 60.1 & 43.0 & 16,436 & - & 4.7 \\
\hline Sub-total Humanities & 6,000 & 21.0 & 58.9 & 40.5 & 16,904 & 62.1 & 16.6 \\
\hline Anthropology & 331 & 1.2 & 61.8 & 38.6 & 16,834 & 41.0 & 17.8 \\
\hline Area Studies & 119 & 0.4 & 62.8 & - & 17,701 & - & - \\
\hline Commerce, Bustaess Administration & 1,051 & 3.7 & 39.1 & 37.4 & 17,727 & 72.1 & 4.6 \\
\hline Econorics & 904 & 3.1 & 63.8 & 38.3 & 18,563 & 63.3 & 4.2 \\
\hline Geography & 609 & 2.1 & 66.7 & 37.4 & 17,250 & 53.7 & 3.6 \\
\hline Law & 504 & 1.8 & 16.5 & 35.5 & 19,007 & 77.1 & 5.4 \\
\hline Polltical Science & 691 & 2.4 & 58.5 & 38.0 & 17,736 & 64.4 & 7.4 \\
\hline Psychology & 1,229 & 4.3 & 77.0 & 37.2 & 17,173 & 58.2 & 15.9 \\
\hline Social work & 289 & 1.0 & 23.4 & 42.6 & 17,917 & 81.3 & 33.2 \\
\hline Sociology & 848 & 3.0 & 55.7 & 38.1 & 16,491 & 55.5 & 14.7 \\
\hline Sub-total Soc1al Sciences & 6,575 & 23.0 & 55.4 & 37.9 & 17,607 & 62.3 & 10.2 \\
\hline
\end{tabular}


Iable 7 (con'c)

Characteristics of Untversity Teachers by Discipline, 1973-74

\begin{tabular}{|c|c|c|c|c|c|c|c|}
\hline Discipline & Number & Percent & $\begin{array}{l}\text { Percentage } \\
\text { with } \\
\text { Doctorate }\end{array}$ & $\begin{array}{c}\text { Average } \\
\text { Age }\end{array}$ & $\begin{array}{l}\text { Average } \\
\text { Salary }\end{array}$ & $\begin{array}{l}\text { Percentage } \\
\text { Canadian } \\
\text { Citizen }\end{array}$ & $\begin{array}{c}\text { Percentage } \\
\text { Female }\end{array}$ \\
\hline & & & & & $\$$ & & \\
\hline Agriculture & 412 & 1.5 & 79.8 & 43.4 & 19,797 & 80.8 & 3.0 \\
\hline Biology & 697 & 2.4 & 83.1 & 40.6 & 18,767 & 65.8 & 10.5 \\
\hline Botany & 191 & 0.7 & 89.2 & 40.6 & 18,367 & - & 11.9 \\
\hline Household Science \& Related & 238 & 0.8 & 38.9 & 41.0 & 16,087 & - & 77.8 \\
\hline Veterlnary Medicine \& Sclences & 135 & 0.5 & 40.7 & 37.2 & 17,567 & - & 5.0 \\
\hline $200 \log y$ & 315 & 1.1 & 89.2 & 40.1 & 18,391 & 61.4 & 8.7 \\
\hline Sub-total Blological Sciences & 1,988 & 7.0 & 76.1 & 40.8 & 18,468 & 69.6 & 15.7 \\
\hline Architecture & 188 & 0.7 & 7.7 & 40.1 & 17,474 & _- & 3.8 \\
\hline Chemical Engineering & 241 & 0.8 & 87.2 & 40.5 & 20,231 & - & 0.4 \\
\hline Civil Engineering & 444 & 1.6 & 57.8 & 41.1 & 19,512 & - & 0.4 \\
\hline Electrical Engineering & 286 & 1.0 & 72.7 & 40.2 & 19,600 & - & 0.7 \\
\hline Mechanical Engineering & 331 & 1.1 & 63.6 & 40.9 & 19,629 & - & 0.3 \\
\hline Mining Englneering & 109 & 0.4 & 72.6 & 41.3 & 19,943 & - & 0.9 \\
\hline Forestry & 81 & 0.3 & 50.6 & 40.2 & 18,434 & - & 0.3 \\
\hline Other Applied Sciences & 502 & 1.7 & - & - & - & - & - \\
\hline Sub-total Applied Sclences & 2,182 & 7.6 & 59.7 & 40.6 & 19,175 & 72.3 & 0.7 \\
\hline Dentistry & 260 & 0.9 & 18.7 & 41.4 & 22,201 & - & 9.2 \\
\hline Medicine & 3,032 & 10.6 & 42.7 & 41.8 & 21,745 & - & 11.1 \\
\hline Nursing & 431 & 1.5 & 3.9 & 39.1 & 13,333 & - & 98.7 \\
\hline Pharmacy & 143 & 0.5 & 79.6 & 40.8 & 18,873 & - & 10.2 \\
\hline Sub-total Health Professions & 3,846 & 13.5 & 38.2 & 41.4 & 20,764 & 73.4 & 20.6 \\
\hline Mathematics & 1,315 & 3.9 & 78.2 & 37.8 & 18,016 & 57.3 & 5.3 \\
\hline Chemistry & 1,162 & 3.5 & 90.3 & 39.9 & 19,449 & 66.4 & 5.7 \\
\hline Geology and Related & 516 & 1.5 & 86.9 & 40.1 & 19,108 & 66.4 & 1.4 \\
\hline Physics & 1,124 & 3.9 & 86.6 & 38.6 & 18,383 & 69.3 & 3.0 \\
\hline Sub-total Physical Sciences & 4,218 & 14.8 & 82.0 & 38.9 & 18,618 & 63.2 & 4.1 \\
\hline GRAND TOTAL & 28,539 & 100.0 & 56.8 & 40.0 & 18,369 & 66.3 & 13.0 \\
\hline
\end{tabular}


Employment of Ph.Ds by Year of Appointment and Discipline in Federal Departments under the Public Service Employment Act*, 1940 to 1972

\begin{tabular}{|c|c|c|c|c|c|c|c|c|c|c|c|c|c|c|c|c|c|c|}
\hline & Before 1940 & $1940-49$ & $1950-54$ & $1954-59$ & 1960 & 1961 & 1962 & 1963 & 1964 & 1965 & 1966 & 1967 & 1968 & 1969 & 1970 & 1971 & 1972 & Total \\
\hline Humanities & 5 & 14 & 15 & 14 & 2 & 1 & 3 & 4 & 1 & 3 & 3 & 4 & 3 & 11 & 7 & 7 & 12 & 109 \\
\hline \multicolumn{19}{|l|}{ Soctal Sclences } \\
\hline Economics & - & 3 & 10 & 11 & 2 & 3 & 3 & 1 & 4 & 3 & 2 & 8 & 10 & 4 & 14 & 15 & 11 & 104 \\
\hline Sociology \& Anthropoly & 1 & 1 & - & 1 & 2 & 1 & - & 1 & 1 & 1 & 1 & 3 & 2 & 2 & 4 & 2 & 2 & 25 \\
\hline Political Science & 1 & 2 & 3 & 3 & 1 & - & 1 & - & 1 & 1 & 3 & 1 & 1 & 2 & 1 & - & - & 21 \\
\hline Psychology & - & 1 & 2 & - & - & - & - & - & 1 & - & 1 & 1 & 2 & 3 & 3 & 1 & - & 15 \\
\hline Sub-total Human Sciences & 7 & 21 & 30 & 29 & 7 & 5 & 7 & 6 & 8 & 8 & 10 & 17 & 18 & 22 & 29 & 25 & 25 & 274 \\
\hline \multicolumn{19}{|l|}{ Agricultural \& Blological } \\
\hline Sciences & 26 & 135 & 136 & 120 & 21 & 21 & 30 & 12 & 28 & 54 & 66 & 57 & 49 & 31 & 25 & 23 & 13 & 847 \\
\hline Engineering & 1 & 4 & 1 & 9 & 3 & 5 & 4 & 2 & 6 & 9 & 9 & 6 & 9 & 7 & 8 & 4 & 5 & 92 \\
\hline \multicolumn{19}{|l|}{ Physical Sciences } \\
\hline Chemistry & 3 & 24 & 34 & 31 & 9 & 5 & 7 & 4 & 12 & 28 & 16 & 28 & 24 & 24 & 19 & 13 & 4 & 285 \\
\hline Geology \& Related & 1 & 20 & 34 & 24 & 9 & 10 & 11 & 4 & 9 & 25 & 10 & 20 & 9 & 14 & 6 & 10 & 9 & 215 \\
\hline Mathematics \& Physics & 1 & 9 & 16 & 9 & 7 & 2 & 2 & 5 & 5 & 5 & 8 & 8 & 9 & 13 & 17 & 6 & 5 & 127 \\
\hline \multicolumn{19}{|l|}{ Health Sclences } \\
\hline Dent1stry & - & 1 & 2 & 3 & 2 & 1 & 2 & 1 & 1 & 3 & 3 & 2 & 3 & 2 & 2 & 1 & - & 29 \\
\hline ledicine & - & 16 & 20 & 32 & 2 & 5 & 7 & 6 & 4 & 8 & 12 & 5 & 5 & 6 & 6 & 3 & 3 & 140 \\
\hline Veterinary & 10 & 44 & 61 & 41 & 7 & 10 & 7 & 8 & 13 & 10 & 14 & 8 & 18 & 16 & 8 & 4 & 4 & 283 \\
\hline Sub-total Natural Sciences & 42 & 253 & 304 & 269 & 60 & 59 & 70 & 42 & 78 & 133 & 138 & 134 & 126 & 113 & 91 & 64 & 43 & 2,019 \\
\hline Grand Total & 49 & 274 & 334 & 298 & 67 & 64 & 77 & 48 & 86 & 141 & 148 & 151 & 144 & 135 & 120 & 89 & 68 & 2,293 \\
\hline
\end{tabular}

* Excludes National Research Council, Defense Research Board and all crown corporations

Source: Adapted from a Table prepared by Dr. Valerte Sonnenfeld from unpublished Public Service Commission data. 
During the sixties, about $100 \mathrm{Ph} . \mathrm{Ds}$ joined the federal public service annually. The number fell to 89 in 1971, and 68 in 1972.

In 1972, the overwhelming majority of the Ph.Ds employed by the government had obtained their degrees in the natural sciences: $2,019(88 \%)$. The humanities and social sciences accounted for the remaining $12 \%$.

Table 9 shows employment sectors of Ph.Ds immediately after graduation. In the early seventies, a very small percentage of the graduates in the humanities were employed by government. The percentage in the social sciences was somewhat higher, mainly due to economists. Fewer than $15 \%$ of the physical and applied scientists, who represented the largest group of $\mathrm{Ph}$.Ds produced, joined the government during these years.

\section{Unemployment - Under - utilization}

Table 9 also shows the unemployment rate of Ph.Ds by field of study. The rates indicate that only a small number are actually unemployed; a more critical question is whether they obtain positions in which their training is effectively utilized. By virtue of their education, aptitude and motivation, Ph.D. graduates are able to displace masters and bachelors degree-holders. Under-utilization is more the issue than unemployment.

This topic has not received the attention it deserves. In recent years, one-third of the $\mathrm{Ph} . \mathrm{D}$. graduates in the natural sciences have continued their training as post-doctorals. It has been estimated that between 2,000 and 3,000 $\mathrm{Ph}$.Ds are now engaged in postdoctoral studies, many of them in a kind of holding pattern, since viable employment opportunities are scarce.

\section{Degrees}

From 1960-61 to 1973-74, Canadian universities awarded 14,280 Ph.Ds, $60 \%$ of them between 1969-70 and 1973-74. The annual number increased from 300 during the early sixties, to almost 2,000 in the early seventies.

Table 10 shows the number of doctoral degrees awarded between 1960-61 and 1973-74 by broad field of study, and Table 11 gives the same information for selected disciplines. For the 14-year period, annual Ph.D. output in education increased from 7 to 120 , and in engineering from 19 to 300 . The number of Ph.Ds in mathematics rose from 10 to 150 , and in psychology, from 20 to 150 . It should also be remembered that in those years, a large number of Canadians obtained doctoral degrees abroad, particularly in the human sciences, and most of them returned to Canada.

During the sixties, almost three-quarters of the $\mathrm{Ph}$.Ds awarded by Canadian universities were in the natural sciences, but this proportion has declined to two-thirds. The humanities and social sciences represent only $33 \%$ of Ph.D. output, although close to $60 \%$ of doctoral enrolment. This reflects a longer completion time, and a higher withdrawal rate.

Between 1964-65 and 1971-72, 10,876 new university positions, in addition to replacements, were created in the humanities and social sciences. But Canada produced only 2,627 Ph.Ds in these fields, including foreign students who returned home and graduates who might have accepted employment in industry and government. It is abvious, therefore, that there was a substantial scarcity of teachers with a Ph.D. Universities' short-term remedies were to hire landed immigrants, and lower the formal teaching qualifications. These practices had two results: 1) the proportion of foreign- 
Table 9

Employment Sector of $\mathrm{Ph}$.Ds Immediately after Graduation from Canadian Universities by Field of Study, 1970-71 to $1974-75$

\begin{tabular}{|c|c|c|c|c|c|c|c|c|c|c|}
\hline & \multicolumn{5}{|c|}{ Wumantities } & \multicolumn{5}{|c|}{ Social Sciences } \\
\hline & $1970-71$ & $1971-72$ & $1972-73$ & $1973-74$ & $1974-75$ & $1970-71$ & $1971-72$ & $1972-73$ & $1973-74$ & $1974-75$ \\
\hline University Teaching & 84.8 & 83.5 & 70.2 & 66.1 & 52.6 & 74.3 & 63.3 & 59.2 & 51.9 & 51.0 \\
\hline Industry & - & - & - & 0.8 & 3.6 & 1.6 & 1.6 & 4.7 & 3.8 & 5.3 \\
\hline Government & 1.9 & 1.7 & 4.9 & 5.5 & 5.8 & 9.3 & 7.6 & 15.4 & 14.6 & 19.0 \\
\hline Private Research Institutes & 1.9 & 1.1 & 2.7 & 2.0 & 4.5 & 2.7 & 4.0 & 3.0 & 6.6 & 5.5 \\
\hline $\begin{array}{l}\text { Other (mostly in the educa- } \\
\text { tional sector) }\end{array}$ & 5.1 & 9.7 & 13.8 & 18.9 & 19.4 & 9.8 & 20.7 & 14.5 & 20.3 & 6.3 \\
\hline Unemployed & 6.3 & 4.0 & 8.4 & 6.7 & 8.1 & 2.2 & 2.8 & 3.2 & 2.7 & 2.9 \\
\hline Total Number & 158 & 176 & 225 & 254 & 222 & 183 & 251 & 338 & 364 & $41 \epsilon$ \\
\hline $\begin{array}{l}\text { Number in Post Doctoral } \\
\text { Studies* }\end{array}$ & 4 & 3 & 5 & 4 & 4 & 14 & 11 & 12 & 16 & 20 \\
\hline
\end{tabular}

* Those Ph.D. graduates who were persuing post doctoral studies have been excluded from the percentage distribution.

Source: Adapted from data of the Canadian Association of Graduate Schools. 
Employment Sector of Ph.Ds Immediately after Graduation from Canadian Universities by Field of Study, $1970-71$ to $1974-75$

\begin{tabular}{|c|c|c|c|c|c|c|c|c|c|c|}
\hline & \multicolumn{5}{|c|}{ Life Sciences } & \multicolumn{5}{|c|}{ Physical and Applied Sciences } \\
\hline & $1970-71$ & $1971-72$ & $1972-73$ & $1973-74$ & $1974-75$ & $1970-71$ & $1971-72$ & $1972-73$ & $1973-74$ & $1974-75$ \\
\hline University Teaching & 46.5 & 40.0 & 40.7 & 28.2 & 27.3 & 41.0 & 38.8 & 33.1 & 31.4 & 31.3 \\
\hline Industry & 8.8 & 6.3 & 5.9 & 9.2 & 11.2 & 22.7 & 25.7 & 29.2 & 30.4 & 22.2 \\
\hline Government & 22.3 & 18.0 & 18.6 & 30.1 & 21.5 & 14.7 & 12.9 & 13.2 & 14.3 & 14.8 \\
\hline Private Research Institutes & 5.3 & 9.8 & 16.6 & 14.5 & $1 \varepsilon .5$ & 1.9 & 4.8 & 9.2 & 10.2 & 12.5 \\
\hline $\begin{array}{l}\text { Other (mostly in the educa- } \\
\text { tion sector) }\end{array}$ & 9,4 & 21.0 & 12.2 & 15.3 & 14.1 & 9.1 & 10.9 & 11.3 & 9.1 & 11.7 \\
\hline Unemployed & 7.6 & 4.9 & 5.9 & 2.7 & 7.3 & 10.5 & 6.9 & 4.0 & 4.6 & 7.4 \\
\hline Total Number & 170 & 205 & 253 & 262 & 205 & 427 & 420 & 469 & 461 & 351 \\
\hline $\begin{array}{l}\text { Number in Post Doctoral } \\
\text { Studies* }\end{array}$ & 126 & 131 & 160 & 152 & 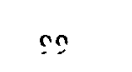 & 232 & 249 & 277 & 244 & 164 \\
\hline
\end{tabular}

* Those Ph.D. graduates who were persuing post doctoral studies have been excluded from the percentage distribution.

Source: Adapted from data of the Canadian Association of Graduate Schools 
Table 10

Doctoral Degrees Awarded by Fleld of Study, 1960-61 to 1973-74

\begin{tabular}{|c|c|c|c|c|c|c|c|c|c|c|c|c|c|c|}
\hline & $1960-61$ & $1961-62$ & $1962-63$ & $1963-64$ & $1964-65$ & $1965-66$ & $1966-67$ & $1967-68$ & $1968-69$ & $1969-70$ & $1970-71$ & $1971-72$ & $1972-73$ & $1973-74$ \\
\hline Humanities & 57 & 42 & 57 & 52 & 74 & 87 & 94 & 96 & 119 & 157 & 188 & 208 & 231 & 235 \\
\hline Soctal sctences & 41 & 35 & 39 & 56 & 56 & 70 & 78 & 134 & 157 & 166 & 229 & 231 & 291 & 290 \\
\hline Education & 7 & 17 & 12 & 13 & 22 & 25 & 39 & 73 & 60 & 78 & 77 & 109 & 123 & 120 \\
\hline Sub-total Human Sclences & 105 & 94 & 108 & 121 & 152 & 182 & 211 & 303 & 336 & 401 & 494 & 548 & 645 & 645 \\
\hline Percentage Human Sctences* & $(34)$ & (29) & $(26)$ & (25) & (27) & (26) & (27) & (30) & (30) & (29) & (30) & (32) & (33) & (33) \\
\hline $\begin{array}{l}\text { Agriculture and Biological } \\
\text { Sciences }\end{array}$ & 57 & 67 & 100 & 99 & 97 & 125 & 115 & 154 & 173 & 235 & 276 & 240 & 249 & 250 \\
\hline $\begin{array}{l}\text { Engineering and applied } \\
\text { Sclences }\end{array}$ & 19 & 20 & 26 & 46 & 45 & 83 & 105 & 103 & 168 & 188 & 225 & 261 & 300 & 300 \\
\hline $\begin{array}{l}\text { Health Professions and } \\
\text { Occupations }\end{array}$ & 24 & 25 & 30 & 31 & 44 & 46 & 50 & 58 & 56 & 95 & 102 & 151 & 180 & 185 \\
\hline $\begin{array}{l}\text { Mathematics and Physical } \\
\text { Sciences }\end{array}$ & 101 & 115 & 157 & 184 & 228 & 260 & 298 & 388 & 375 & 456 & 528 & 524 & 558 & 560 \\
\hline Sub-total Natural Sclences & 201 & 227 & 313 & 360 & 414 & 514 & 568 & 703 & 772 & 974 & 1,131 & 1,176 & 1,287 & 1,295 \\
\hline Percentage Natural Sciences* & (66) & (71) & (74) & $(75)$ & (73) & $(74)$ & (73) & $(70)$ & $(70)$ & (71) & $(70)$ & (68) & $(67)$ & (67) \\
\hline Grand Total & 306 & 321 & 421 & 481 & 566 & 696 & 779 & 1,006 & 1,108 & 1,375 & 1,625 & 1,724 & 1,932 & 1,940 \\
\hline
\end{tabular}

* Percentage breakdown between Human Sclences and Natural Sclences in brackets.

Source: Statistics Canada. 
Table 11

Doctoral Degrees Awarded By Selected Disciplines, 1960-61 to 1972-73

\begin{tabular}{|c|c|c|c|c|c|c|c|c|c|c|}
\hline & $\begin{array}{l}\text { Fine } \\
\text { Arts }\end{array}$ & $\begin{array}{c}\text { Economics } \\
\& \\
\text { Business }\end{array}$ & Geography & $\begin{array}{c}\text { Political } \\
\text { Science }\end{array}$ & Psychology & $\begin{array}{l}\text { Soc1a1 } \\
\text { Work }\end{array}$ & Agriculture & Medec1ne & Mathematics & $\begin{array}{c}\text { Chemistry } \\
\& \\
\text { Physics }\end{array}$ \\
\hline $1960-61$ & - & 10 & 2 & 2 & 25 & - & 6 & 23 & 8 & 81 \\
\hline $1961-62$ & - & 5 & 3 & 1 & 20 & 1 & 16 & 25 & 10 & 93 \\
\hline $1962-63$ & - & 6 & 3 & 2 & 24 & 1 & 17 & 30 & 6 & 135 \\
\hline $1963-64$ & 2 & 7 & 3 & 2 & 35 & - & 17 & 27 & 21 & 142 \\
\hline $1964-65$ & 1 & 12 & 5 & - & 31 & 3 & 11 & 41 & 28 & 159 \\
\hline $1965-66$ & 1 & 14 & 3 & 5 & 44 & - & 16 & 40 & 34 & 177 \\
\hline $1966-67$ & 2 & 10 & 8 & 1 & 47 & - & 14 & 46 & 43 & 203 \\
\hline $1967-68$ & - & 20 & 10 & 10 & 82 & 3 & 15 & 52 & 49 & 276 \\
\hline $1968-69$ & - & 21 & 13 & 8 & 98 & 2 & 27 & 50 & 53 & 282 \\
\hline $1969-70$ & 3 & 15 & 14 & 18 & 86 & 2 & 60 & 87 & 61 & 332 \\
\hline $1970-71$ & 6 & 28 & 18 & 21 & 119 & 1 & 46 & 95 & 85 & 369 \\
\hline $1971-72$ & 6 & 27 & .22 & 31 & 109 & 1 & 52 & 134 & 97 & 356 \\
\hline $1972-73$ & 5 & 49 & 24 & 20 & 121 & 6 & 64 & 163 & 113 & 375 \\
\hline Tota1 & 26 & 224 & 128 & 121 & 841 & 20 & 361 & 813 & 608 & 2,980 \\
\hline
\end{tabular}

Source: Statistics Canada 
FULI, AND PART-TTME DOCTORAL, STUDENT FNROLMENT

BY FIELD OF STUDY, 1969-70 TO 1975-76

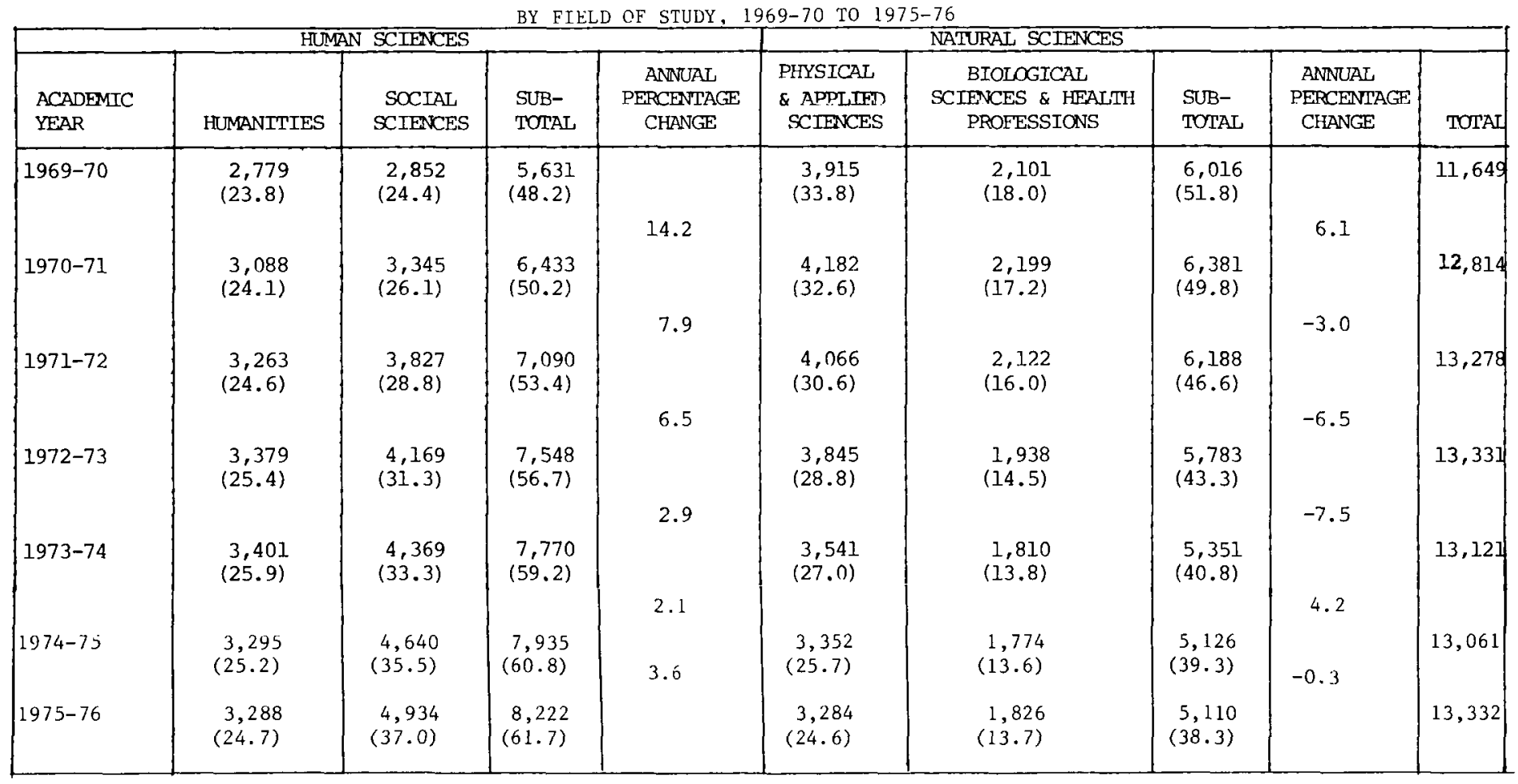

*Figures in brackets indicate the percentage by field of study.

Source: Adapted from the Canadian Association of Graduate Schools data. 
born university teachers increased rapidly for a number of years, a situation which had implications for Canada's cultural identity, and 2) some who were hired might have been better suited to other activities

\section{PH.D. ENROLMENT AND GOVERNMENT ASSISTANCE}

\section{Numbers}

Doctoral enrolment trends form the basis of the future supply of Ph.Ds. In the early seventies, about 13,000 full- and part-time students were enrolled at Canadian universities. Unlike the sixties when enrolment increased rapidly each year, between 1970-71 and 1974-75 it levelled off but increased in 1975-76. The proportion of part-time doctoral students rose from 19.7\% of full-time enrolment in 1969-70 to $33.6 \%$ in 1975-76.

There was a marked shift during the early seventies from the natural sciences to the humanities and social sciences. In 1969-70, the former accounted for $51.8 \%$ of all doctoral students, but the percentage declined to $38.3 \%$ in $1975-76$ (Table 12). Conversely, the humanities and social sciences increased from $48.2 \%$ to $61.7 \%$. In absolute numbers, enrolment in the physical and applied sciences fell from 3,915 to 3,284 , whereas it rose from 2,852 to 4,934 in the social sciences, and has remained constant in the humanities and life sciences.

\section{Geographic Location}

Another important feature of Canadian doctoral enrolment is the fact that more than $50 \%$ of it is in Ontario universities. The University of Toronto alone enrolled more than $20 \%$ of all doctoral students (Table 13).

\section{Citizenship}

Expansion of Canadian graduate education at the doctoral level was achieved, to a large extent, with foreigh-born graduate students frequently taught by foreign-born faculty. A large percentage of full-time doctoral students are non-Canadian (Table 14). In 1972-73, landed immigrants accounted for almost one-third, while another $15 \%$ were foreign students. Table 15 shows that Americans were the largest single group from abroad, constituting $12.6 \%$ of all enrolment, with a high of $22.7 \%$ in the humanities and a low of $2.6 \%$ in engineering. In contrast, doctoral students from Asian countries made up $13.3 \%$, with a low of $2.7 \%$ in the humanities and a high of $32.5 \%$ in engineering.

\section{Government Assistance}

A possible contributing factor for the increased number of foreign-born doctoral students was the formula financing scheme in some provinces, which allocated funds to universities on a per-capita of enrolment basis. By 1975-76, Ontario universities were receiving about $\$ 12,000$ a year from the provincial government, for each Ph.D. student, in addition to tuition fees. Thus, it was in the universities' interest to expand doctoral enrolment. Moreover, there were support programs for graduate students.

Most doctoral students at Canadian universities have been supported by federal or provincial government fellowships, by teaching or research assistantships and scholarships 
FULU, AND PART-TTME DOCTORAL ENROLMENT AT FTVE SETECTED

UNIVERSITIES* $1968-69$ TO 1975-76

\begin{tabular}{|c|c|c|c|c|c|c|c|c|}
\hline YEAR & ALBERTA & $\begin{array}{l}\text { BRT'ISH } \\
\text { COLUMBIA }\end{array}$ & MCGIII & MONTREAL & TORONTO & $\begin{array}{l}\text { SUB- } \\
\text { TOTAL }\end{array}$ & $\begin{array}{l}\text { OMHER } 22 \\
\text { UNTVERSITIES }\end{array}$ & TOTAL \\
\hline $1968-69$ & $\begin{array}{r}808 \\
(8.4)\end{array}$ & $\begin{array}{r}882 \\
(9.2)\end{array}$ & $\begin{array}{l}1,016 \\
(10.6)\end{array}$ & $\begin{array}{r}763 \\
(7.9)\end{array}$ & $\begin{array}{l}1,817 \\
(18.9)\end{array}$ & $\begin{array}{c}5,286 \\
(55.0)\end{array}$ & $\begin{array}{c}4,318 \\
(45.0)\end{array}$ & 9,604 \\
\hline $1969-70$ & $\begin{array}{r}961 \\
(8.2)\end{array}$ & $\begin{array}{l}1,015 \\
(8.7)\end{array}$ & $\begin{array}{l}1,327 \\
(11.4)\end{array}$ & $\begin{array}{r}883 \\
(7.6)\end{array}$ & $\begin{array}{l}2,290 \\
(19.6)\end{array}$ & $\begin{array}{c}6,476 \\
(55.5)\end{array}$ & $\begin{array}{l}5,201 \\
(44.5)\end{array}$ & 11,677 \\
\hline $1970-71$ & $\begin{array}{l}1,074 \\
(8.4)\end{array}$ & $\begin{array}{l}1,079 \\
(8.4)\end{array}$ & $\begin{array}{l}1,325 \\
(10.3)\end{array}$ & $\begin{array}{r}973 \\
(7.6)\end{array}$ & $\begin{array}{l}2,550 \\
(19.9)\end{array}$ & $\begin{array}{r}7,001 \\
(54.6)\end{array}$ & $\begin{array}{r}5,813 \\
(45.4)\end{array}$ & 12,814 \\
\hline $1971-72$ & $\begin{array}{l}1,077 \\
(8.1)\end{array}$ & $\begin{array}{l}1,061 \\
(8.0)\end{array}$ & $\begin{array}{l}1,314 \\
(9.9)\end{array}$ & $\begin{array}{l}1,000 \\
(7.5)\end{array}$ & $\begin{array}{c}2,647 \\
(20.0)\end{array}$ & $\begin{array}{r}7,099 \\
(53.5)\end{array}$ & $\begin{array}{c}6,169 \\
(46.5)\end{array}$ & 13,268 \\
\hline $1972-73$ & $\begin{array}{l}1,019 \\
(7.6)\end{array}$ & $\begin{array}{c}1,024 \\
(7.7)\end{array}$ & $\begin{array}{l}1,239 \\
(9.3)\end{array}$ & $\begin{array}{c}1,116 \\
(8.4)\end{array}$ & $\begin{array}{l}2,700 \\
(20.3)\end{array}$ & $\begin{array}{c}7,098 \\
(53.2)\end{array}$ & $\begin{array}{c}6,233 \\
(46.8)\end{array}$ & 13,331 \\
\hline $1973-74$ & $\begin{array}{r}942 \\
(7.2)\end{array}$ & $\begin{array}{r}948 \\
(7.2)\end{array}$ & $\begin{array}{l}1,202 \\
(9.2)\end{array}$ & $\begin{array}{l}1,174 \\
(9.0)\end{array}$ & $\begin{array}{c}2,724 \\
(20.8)\end{array}$ & $\begin{array}{c}6,990 \\
(53.3)\end{array}$ & $\begin{array}{c}6,131 \\
(46.7)\end{array}$ & 13,121 \\
\hline $1974-75$ & $\begin{array}{r}910 \\
(7.0)\end{array}$ & $\begin{array}{r}891 \\
(6.8)\end{array}$ & $\begin{array}{l}1,128 \\
(8.6)\end{array}$ & $\begin{array}{l}1,174 \\
(9.0)\end{array}$ & $\begin{array}{r}2,854 \\
(21.9)\end{array}$ & $\begin{array}{r}6,957 \\
(53.3)\end{array}$ & $\begin{array}{r}6,104 \\
(46.7)\end{array}$ & 13,061 \\
\hline $1975-76$ & $\begin{array}{r}895 \\
(6.7)\end{array}$ & $\begin{array}{r}836 \\
(6.3)\end{array}$ & $\begin{array}{l}1,082 \\
(8.1)\end{array}$ & $\begin{array}{r}1,372 \\
(10.3)\end{array}$ & $\begin{array}{r}2,821 \\
(21.2)\end{array}$ & $\begin{array}{r}7,006 \\
(52.6)\end{array}$ & $\begin{array}{c}6326 \\
(47.4)\end{array}$ & 13,332 \\
\hline
\end{tabular}

*Figures in brackets indicate percentage distribution.

Source: Adapted from the Canadian Association of Graduate Schools data. 
CITIZENSHIP AND IMMTGRATTON STATUS OF FULI-TIME MASTERS AND PH.D.

STUDENTS BY FIFID OF STUDY, 1972-1973

(in percent)

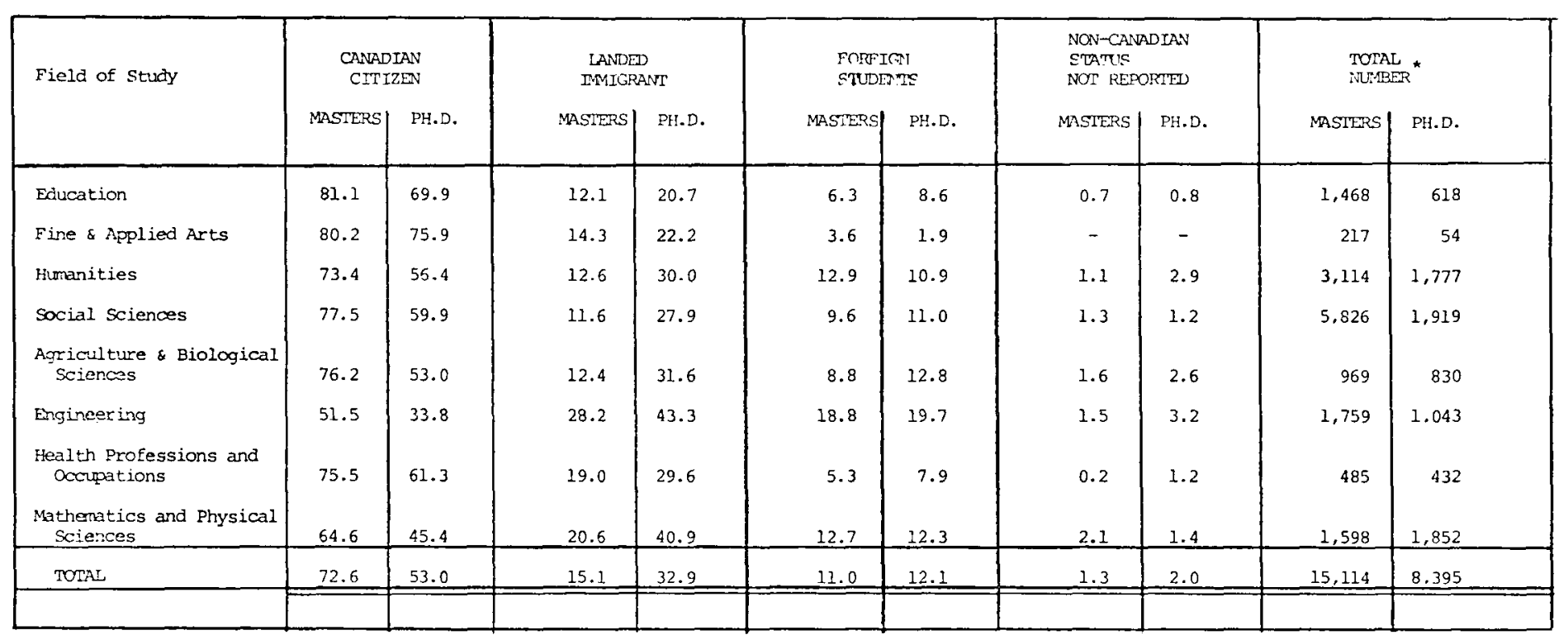

* The legal status of graduate students was available for only ahout 8 n: of the master's and doctoral students.

Source: Statistics Canada, unpublished data. 
CITIZENSHIP OF FULL-TIME PH.D. STUDENTS BY COUTTPY ART FIFID OF SIUDY, 1972-1973

(in percent)

\begin{tabular}{|c|c|c|c|c|c|c|c|c|c|c|}
\hline FIEID OF STUDY & CANADA & $\begin{array}{l}\text { UNITED } \\
\text { STATES }\end{array}$ & $\begin{array}{l}\text { UNTIED } \\
\text { KINGDOM }\end{array}$ & $\begin{array}{l}\text { FRANCE } \\
\text { \& OTIETR } \\
\text { EUROPEAN }\end{array}$ & CARIBBEAN & $\begin{array}{l}\text { CENTRAL } \\
\& \text { SOUTH } \\
\text { ANERICA }\end{array}$ & AFRICA & $\begin{array}{c}\text { SOUTH } \\
\text { PACIFIC }\end{array}$ & ASIA & $\begin{array}{l}\text { TOTAL } \\
\text { NLMBER }\end{array}$ \\
\hline Edication & 70.5 & 10.8 & 3.6 & 2.1 & 0.9 & 0.2 & 2.4 & 3.9 & 5.7 & 614 \\
\hline Muranities & 57.2 & 22.7 & 6.8 & 5.7 & 1.0 & 0.6 & 1.4 & 1.9 & 2.7 & 1,751 \\
\hline $\begin{array}{l}\text { Agriculture and Biological } \\
\text { Sciences }\end{array}$ & 53.9 & 12.0 & 8.2 & 4.2 & 0.7 & 0.6 & 2.7 & 2.6 & 15.2 & 817 \\
\hline Engineering & 34.7 & 2.6 & 4.2 & 10.1 & 0.3 & 2.0 & 11.8 & 1.8 & 32.5 & 1,017 \\
\hline $\begin{array}{l}\text { Health Professions and } \\
\text { occupations }\end{array}$ & 61.6 & 4.8 & 3.6 & 7.4 & 1.4 & 1.5 & 1.1 & 1.7 & 17.0 & 927 \\
\hline $\begin{array}{l}\text { Mathematics and Physical } \\
\text { Sciences }\end{array}$ & 46.8 & 8.0 & 9.6 & 7.0 & 0.6 & 1.5 & 2.0 & 2.8 & 21.7 & 1,798 \\
\hline TOTAL & 53.8 & 12.6 & 6.9 & 5.9 & 0.7 & 0.9 & 3.5 & 2.4 & 13.3 & 8,442 \\
\hline
\end{tabular}

* The information was available for about 808 of the full-time doctoral students.

Source: Statistics Canada, unpublished data, 
from the universities, or by student loans. It has been estimated that $50 \%$ to $75 \%$ of doctoral students in the physical and applied sciences have obtained funding through National Research Council grants. (The exact number is difficult to determine because National Research Council support could consist of either direct fellowships to students, or research grants provided to universities or individual faculty members, which enable them to hire doctoral students as research assistants.) Consequently, in most of the natural sciences doctoral students have experienced little difficulty obtaining financial support to cover their living and transportation expenses. By contrast, only one-third of the full-time doctoral students in the humanities and social sciences have been supported by the Canada Council. Others have received fellowships from the provinces, or have benefited from the federal Canada Student Loan Plan.

The Canada Council increased the number of fellowships in the humanities and social sciences from 426 in 1965-66 to a high of 2,456 in 1970-71; they declined to 1,387 by 1975-76. Table 16 shows the number of Canada Council doctoral fellowships by discipline between 1965-66 and 1975-76. An estimated one-third of the full-time doctoral students at Canadian universities were benefiting each year from the Council's Program.

The federal Canada Student Loan Plan has made it possible for doctoral students to borrow interest-free $\$ 1,000$ to $\$ 1,800$ annually (up to a present maximum of $\$ 9,800$ ), depending on the province (excluding Quebec), and the year the loan was granted. The percentage who have done so is comparatively low. As an illustration, during the late sixties and early seventies, there were about 10,000 full-time doctoral students at Canadian universities each year, out of which only a few hundred took advantage of the Canada Student Loan Plan. Table 17 gives the number of Canada Student Loan Plan certificates issued, by province: a total of 373 in 1964-65 which increased to 3,238 in 1974-75. The majority, 3035 , went to Ontario residents. The number of certificates in Ontario had risen from 504 in 1971-72 to 2,177 the next year, reflecting a change in the Ontario Student Assistance Program so that it consisted of an $\$ 800$ loan and a grant of up to $\$ 600$.

The Canada Student Loan Plan also offered financial assistance for doctoral studies abroad. In 1967-68, of the 593 recipients, $29.7 \%$ were studying in the United States, $10.6 \%$ in the United Kingdom, and $3 \%$ in other countries (Table 18). Six years later, in 1973-74, the number of loan recipients had increased to 2,656 . However, of the 2,451 studying in Canada, 2,275 were in Ontario. Studies abroad had declined: $4.2 \%$ in the United States, $2.2 \%$ in the United Kingdom, and $1.3 \%$ in other countries.

Since most doctoral students receive financial assistance from universities and federal and provincial sources, their main economic contribution to their education consists of foregone income.

\section{DOCTORAL PROGRAMS}

\section{Historical Development}

At present, 34 universities offer Ph.D. programs, most of which were created during the sixties and early seventies. This is a considerable change from 1944-45 when only five 
TABLE 16

Canada Council Doctoral Fellowship Holders

By Disc1pline, 1965-66 to 1975-76

\begin{tabular}{|c|c|c|c|c|c|c|c|c|c|c|c|}
\hline & $1965-66$ & $1956-67$ & $1967-68$ & $1968-69$ & $1969-70$ & $1970-71$ & $1971-72$ & $1972-73$ & $1973-74$ & $1974-75$ & $1975-76$ \\
\hline Actinistrative studies (I) & - & - & _- & - & 23 & & 31 & 24 & 18 & 24 & 12 \\
\hline $\begin{array}{l}\text { Busiress Ackinistration } \\
\text { B's }\end{array}$ & - & - & - & - & 59 & 64 & 68 & 47 & 36 & 29 & 21 \\
\hline Anthrcpolony (Archecology) & 9 & 36 & 55 & 79 & 86 & 99 & 96 & 90 & 75 & 76 & 74 \\
\hline 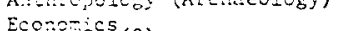 & 53 & 125 & 181 & 234 & 207 & 215 & 182 & 135 & 113 & 95 & 79 \\
\hline $\begin{array}{l}\text { Econatics }(2) \\
\text { Eucazisnt }\end{array}$ & - & - & - & - & 58 & 92 & 93 & 137 & 157 & 144 & 152 \\
\hline 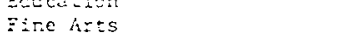 & 19 & 34 & 53 & 59 & 65 & 77 & 88 & 71 & 58 & 64 & 48 \\
\hline $\begin{array}{l}\text { are Arts } \\
\text { Geosrariy a Detography }\end{array}$ & 23 & 31 & 47 & 69 & 54 & 63 & 56 & 46 & 36 & 35 & 30 \\
\hline 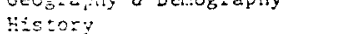 & 68 & 132 & 230 & 303 & 272 & 257 & 261 & 214 & 175 & 149 & 125 \\
\hline $\begin{array}{l}\text { nastory } \\
\text { chassics }\end{array}$ & 13 & $\therefore 2$ & 45 & 48 & 44 & 43 & 33 & 22 & 18 & 19 & 18 \\
\hline $\begin{array}{l}\text { Cassios } \\
\text { Eagizin }\end{array}$ & 52 & 113 & 211 & 321 & 323 & 313 & 286 & 204 & 173 & 136 & 124 \\
\hline $\begin{array}{l}\text { Engtisn } \\
\text { Franci }\end{array}$ & 41 & 68 & 115 & 152 & 144 & 138 & 112 & 91 & 74 & 60 & 49 \\
\hline $\begin{array}{l}\text { Sences } \\
\text { German }\end{array}$ & 6 & 9 & 27 & 40 & 36 & 34 & 29 & 19 & 18 & $2 \mathrm{~L}$ & 19 \\
\hline $\begin{array}{l}\text { ueroan } \\
\text { Otiner Foreign Larguages }\end{array}$ & 10 & 20 & 39 & 56 & 76 & 79 & 86 & 69 & 59 & 54 & 56 \\
\hline $\begin{array}{l}\text { otner foreagn Languages } \\
\text { Lain }\end{array}$ & 3 & 14 & 27 & 51 & 48 & 40 & 26 & 34 & 34 & 31 & 34 \\
\hline Linguistics & 4 & 26 & 41 & 69 & 82 & 68 & 66 & 44 & 42 & 33 & 35 \\
\hline 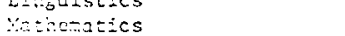 & 2 & 2 & 12 & 16 & 36 & 44 & 44 & 32 & 27 & 19 & 14 \\
\hline 7 Pesosin & 32 & 84 & $15 i$ & 219 & 190 & 183 & 170 & 117 & 100 & 81 & 86 \\
\hline reliticai seience & 37 & 99 & 154 & 184 & 183 & 104 & 183 & 153 & 133 & 122 & 103 \\
\hline Fevolong & 25 & 29 & 51 & .110 & 122 & 167 & 200 & 183 & 175 & 270 & 173 \\
\hline 3eicious studics ${ }^{(3)}$ & - & - & - & - & $\begin{array}{r}122 \\
50\end{array}$ & 55 & 58 & 43 & 40 & 31 & 26 \\
\hline Social fork & - & _ & - & _- & 2 & 4 & 5 & 3 & 3 & - & 78 \\
\hline Socio:? $\approx y$ & 39 & 85 & 115 & 276 & 163 & 165 & 166 & 133 & 120 & 102 & $\begin{array}{l}18 \\
30\end{array}$ \\
\hline other $(6)^{2}$ & - & - & - & - & 45 & 36 & 56 & 42 & 38 & 39 & \\
\hline TOTAL & 426 & 949 & 1,554 & 2,183 & 2,368 & 2,456 & 2,395 & 1,955 & 1,722 & $I, 534$ & 1. 387 \\
\hline
\end{tabular}

* Between 19j7-58 and $1964-65$ a total of only 1,318 predoctoral fellowships were granted: 97 in 1957-58; 110 in 1958-59; 121 1n 1959-60; 133 1n 1960-61; 169 in $1962-62 ; 284$ in $1962-63 ; 216$ in $1963-64 ; 288$ in $1964-65$.

(1) prior to 1959-70, Public Administration was included in Polltical Sclence and Business; and Administration of ten under Economics.

(2) Prior to 1969-70, Education was Included in Psychology.

(3) irior to 2959-70, Reilgious studtes were included in Philosophy.

(4) This category Includes areas such as Urban and Regional Studies, Communication Studies, Criminology, Information Sclences, and Interdisciplinary subjects. Source: Annual keports of tne canatad Cuuntia. 
Canada Student Loan Plan Certificates for Doctoral Students by Province, 1964-65 to 1974-75

\begin{tabular}{|c|c|c|c|c|c|c|c|c|c|c|c|}
\hline & $1964-65$ & $1965-66$ & $1966-67$ & $1967-68$ & $1968-69$ & $1969-70$ & $1970-71$ & $1971-72$ & $1972-73$ & $1973-74$ & $1974-$ \\
\hline Newfoundland & 2 & 5 & 5 & 8 & 11 & 11 & 7 & 7 & 10 & 5 & 3 \\
\hline Prince Edward Island & 1 & 3 & 3 & 1 & 2 & - & 2 & 6 & 6 & 2 & 3 \\
\hline Nova Scotia & 23 & 19 & 9 & 24 & 22 & 32 & 29 & 40 & 27 & 45 & 20 \\
\hline New Brunswick & 8 & 13 & 19 & 26 & 12 & 18 & 11 & 10 & 10 & 6 & 10 \\
\hline Ontario & 244 & 208 & 127 & 258 & 162 & 185 & 348 & 504 & 2,177 & 2,472 & 3,035 \\
\hline Manitoba & 14 & 20 & 34 & 32 & 24 & 35 & 35 & 32 & 36 & 28 & 55 \\
\hline Saskatchewan & 9 & 23 & 23 & 28 & 24 & 30 & 29 & 16 & 28 & 10 & 17 \\
\hline Alberta & 22 & 51 & 71 & 83 & 106 & 132 & 115 & 84 & 63 & 23 & 31 \\
\hline British Columbia & 50 & 98 & 112 & 133 & 91 & 95 & 118 & 72 & 50 & 65 & 64 \\
\hline Total & 373 & 440 & 403 & 593 & 454 & 538 & 694 & 771 & 2,407 & 2,656 & 3,238 \\
\hline
\end{tabular}

Note: The province of Quebec does not particlpate in the Canada Student Loan Plan, but has its own student assistance program.

Source: Department of Finance 
Canada Student Loan Plan Doctoral Student Recipients by Province or Country of Study, 1964-65 to 1974-.75

\begin{tabular}{|c|c|c|c|c|c|c|c|c|c|c|c|}
\hline & $64-65$ & $1965-66$ & $1966-67$ & $1967-68$ & $1963-69$ & $1969-70$ & $1970-71$ & $1971-72$ & $1972-73$ & $1973-74$ & $1974-$ \\
\hline Newfoundland & - & 1 & 1 & 1 & - & 1 & - & 2 & 4 & 7 & 12 \\
\hline Prince Edward Island & $1-$ & - & 1 & - & - & - & - & - & 1 & - & - \\
\hline Nova Scotia & 20 & 12 & 7 & 10 & 8 & 13 & 11 & 19 & 21 & 38 & 13 \\
\hline New Brunswick & 2 & 10 & 8 & 9 & 5 & 6 & 4 & 6 & 5 & 5 & 4 \\
\hline Quebec $^{*}$ & 10 & 11 & 12 & 10 & 9 & 12 & 9 & 11 & 38 & 51 & 88 \\
\hline Ontario & 208 & 131 & 81 & 180 & 105 & 125 & 295 & 392 & 1,992 & 2,275 & 2,739 \\
\hline Manitoba & 2 & 5 & 9 & 10 & 5 & 12 & 14 & 15 & 22 & 7 & 46 \\
\hline Saskatchewan & 5 & 5 & 7 & 12 & 2 & 3 & 7 & 7 & 8 & 5 & 8 \\
\hline Alberta & 13 & 24 & 32 & 46 & 63 & 80 & 70 & 53 & 61 & 31 & 39 \\
\hline British Columbia & 6 & 48 & 51 & 58 & 32 & 34 & 69 & 41 & 27 & 32 & 19 \\
\hline Sub-Total - CANADA & 266 & 247 & 209 & 336 & 229 & 286 & 479 & 546 & 2,179 & 2,451 & 2,968 \\
\hline United States & 64 & 150 & 143 & 176 & 157 & 169 & 140 & 141 & 129 & 112 & 149 \\
\hline United Kingdom & 17 & 29 & 32 & 63 & 52 & 65 & 60 & 62 & 65 & 59 & 61 \\
\hline other & 6 & 14 & 19 & 18 & 16 & 18 & 15 & 22 & 34 & 34 & 60 \\
\hline Total & 373 & 440 & 403 & 593 & 454 & 538 & 694 & 771 & 2,407 & 2,656 & 3,238 \\
\hline
\end{tabular}

* Quebec is not participating in the Canada Student Loan Plan

Source: Department of Finance 


\author{
Table 19 \\ Number of Canadian Universities Offering Master's \\ and Doctoral Degree Programs, 1944-45 to 1974-75
}

\section{Master's degree}

$1944-45$

1946-47

$1950-51$

$1954-55$

1958-59

$1962-63$

$1966-67$

1970-71.

$1974-75$
Doctor of Philosophy

16

19

24

30

34

Source: Association of Universities and Colleges of Canada

Canadian universities had doctoral programs (Table 19). ${ }^{6}$ During the fifties there was little expansion, and fewer than $300 \mathrm{Ph} . \mathrm{Ds}$ were granted each year, most of them in the natural sciences.

The sixties was an era of dramatic increase in the number of doctoral programs. According to the Handbook of the Association of Universities and Colleges, in 1970,851 different doctoral programs were in operation at 30 universities. By 1974 the number had increased to 1,146 (in addition to 2,000 masters programs), many of which have small enrolments (Table 20). Every province but Prince Edward Island, developed its own programs, without national planning or co-ordination.

Thus, 26 universities have doctoral programs in chemistry, 18 in English literature, 19 in history, 15 in geography, and 12 in sociology. Considering the many options in each discipline, the number of courses is very large.

This is illustrated by a subject like English Literature in which a student can specialize in areas ranging from Medieval studies to modern drama or poetry.

Chemistry, too, is divided into many sub-groups within the major branches. Although some specialties are in demand, a substantial number of doctorates are still produced in

\footnotetext{
${ }^{6}$ Some of these graduate programs are given in affiliation with other universities.
} 
Table 20

Number of Doctoral Programs at Canadian Universities by Discipline, 1974-75

Discipline

Number

Humanities

Fine and Applied Arts

Classics

History

English

French

German

Spanish

Other Modern Languages

Philosophy

Religious Studies

Other humanities

Sub-Total Humanities

\section{Social Sciences}

$\begin{array}{lr}\text { Archaeology } & 7 \\ \text { Anthropology } & 8 \\ \text { Area Studies } & 45 \\ \text { Commerce and Business Administration } & 23 \\ \text { Economics } & 19 \\ \text { Education } & 84 \\ \text { Geography } & 27 \\ \text { Law } & 6 \\ \text { Political Science } & 19 \\ \text { Psychology } & 27 \\ \text { Social Work } & 4 \\ \text { Sociology } & 13 \\ \text { Sub-Total Social Sciences } & 282\end{array}$

\section{Biological Sciences}

Agriculture $\quad 67$

Biology 62

Botany 26

Household Science and related 12

Veterinary Medicine and Science 15

Zoology 8

Other Biological Sciences $\quad 14$

Sub-Total Biological Sciences. 204 


\section{Table 20 (con't)}

Discipline

Number

Applied Sciences

Architecture

Chemical Engineering

21

Civil Eng ineering

Electrical Engineering

20

Mechanical Engineering

Mining Engineering

Forestry

Other Engineering and Applied Sciences

Sub-Total Applied Sciences

\section{Medica1 Sciences}

Dentistry

Medicine

Pharmacy

Other Medical Sciences

Sub-Total Medical Sciences

Physical Sciences

Mathematics

Chemistry

Geology and related

Physics

Other Physical Sciences

Sub-total Physical Sciences

GRAND TOTAL

other disciplines where demand is subsiding. Therefore, shortages and surpluses can exist within one discipline. Since, for economic, political and structural reasons, Canada's chemical industry will not expand substantially, the question of how many universities should offer doctoral programs in chemistry has been raised.

A similar situation seems to have developed in engineering. Altogether, there are 216 different doctoral programs, including 21 in chemical engineering, 17 in civil, 20 in electrical and 18 in mechanical. Because Canadian industry has not hired many engineering Ph.Ds, positions are scarce; for lack of employment opportunities, some students have undertaken post-doctoral studies.

In the past, most Ph.Ds were employed in the university sector and others joined the 
RATIO BETWEEN PH.D. ENROLMENT AND PH.D. AWARDS BY FIETD OF STUDY,

1969-70 To 1974-75 (in percent)

\begin{tabular}{|c|c|c|c|c|c|c|c|c|}
\hline & $1969-70$ & $1970-71$ & $1971-72$ & $1972-73$ & $1973-74$ & $1974-75$ & $\begin{array}{l}\text { SIX YEAR } \\
\text { AVERAGE }\end{array}$ & $\begin{array}{l}\text { TOTAL DEGREES } \\
\text { GRANTED }\end{array}$ \\
\hline Education & 11.6 & 8.3 & 11.0 & 9.9 & 10.8 & 7.8 & 9.9 & 599 \\
\hline Humanities & 4.8 & 4.8 & 6.2 & 7.7 & 7.9 & 8.9 & 6.7 & 1,248 \\
\hline Social Sciences & 7.4 & 6.2 & 7.1 & 7.2 & 8.6 & 8.5 & 7.5 & 1,355 \\
\hline Biological Sciences & 12.9 & 16.6 & 17.2 & 20.3 & 22.2 & 20.4 & 18.3 & 1,117 \\
\hline Applied Sciences & 12.0 & 16.6 & 16.3 & 20.6 & 23.2 & 23.3 & 18.7 & 1,455 \\
\hline Health Occupations & 15.3 & 17.6 & 21.0 & 25.0 & 28.1 & 23.0 & 21.7 & 1,176 \\
\hline Physical Sciences & 15.1 & 15.3 & 20.1 & 19.6 & 24.3 & 21.3 & 19.3 & 2,933 \\
\hline AIL FIEIDS & 10.5 & 11.0 & 12.7 & 13.4 & 15.0 & 13.8 & 12.7 & 9,883 \\
\hline
\end{tabular}

Source: Derived from the Canadian Association of Graduate Schools' data. 
government. The number of openings in these areas has diminished in recent years, and indications are that future job prospects are less than promising.

The present saturation of universities and government is particularly critical for humanities and most social science Ph.Ds; up to $90 \%$ of them were traditionally employed in these sectors, although actual numbers are small. Yet, in 1974-75, there were 131 different doctoral programs in the humanities and 282 in the social sciences.

\section{The Ratio of Degrees to Enrolment}

As a measure of output, the ratio of Ph.Ds granted to total enrolment for a six-year period has been estimated by field of study. To overcome the effect of yearly fluctuations, these calculations were based on a six-year average. Table 21 shows that about $20 \%$ of Ph.D. students have graduated each year in the natural sciences, compared with only $6.7 \%$ in the humanities and $7.5 \%$ in the social sciences.

In chemistry, for example, $23.3 \%$ received doctorates each year compared with $5.2 \%$ in political science and $5.5 \%$ in sociology (Table 22). Expressed differently, it would take a cohort of 100 chemistry doctoral students slightly more than four years to graduate, whereas similar cohorts in political science and sociology would take about 20 years. In absolute numbers, $204 \mathrm{Ph}$.Ds in chemistry were awarded each year between 1969-70 and $1975-76$, but only 23 in political science and 20 in sociology.

A number of illustrations are of interest. Cumulatively, for a seven year period, there were 4,797 Ph.D. students in physics and 958 degrees were granted - $137(20.0 \%)$ each year. Enrolment in English was greater - 5,630 students - but only $409 \mathrm{Ph}$.Ds or 58 (7.3\%) a year were granted.

The ratio of enrolment to $\mathrm{Ph} . \mathrm{D}$. awards in most of the humanities and social sciences requires thoughtful analysis. More careful selection of students might lessen the drop-out rate $(50 \%)$ and reduce the length of time for completion of a doctorate. Although the formal requirement from masters or equivalent standing to a Ph.D. is two to three years, the normal time is five years. By contrast, in most sciences the actual length of study is three years, with a withdrawal rate of less than $25 \%$.

Differences in Ph.D. productivity have been attributed to the less formal structure of the humanities and social sciences, greater emphasis on the dissertation, and the newness of many doctoral programs. Whatever the reason, there is a need for change. From a student's point of view, an indefinite period of study is frustrating and costly, and from society's vantage point, it is also expensive. As previously mentioned, most provinces pay universities more than $\$ 10,000$ annually for each Ph.D. student.

But the small number of doctorates conferred in most of the humanities and social sciences in relation to Ph.D. enrolment in those disciplines may have been a blessing in disguise for the seventies. Otherwise, the number of Ph.Ds seeking employment would have been even larger.

\section{The Irregular Supply of Ph.D.s}

A cycle of shortage and surplus in the supply of Ph.D.s appears to be developing in some disciplines. In 1973-74 and 1974-75, fewer new doctoral students registered, than there were Ph.D.s granted. Only 1,545 new doctoral students enrolled in 1973-74, whereas 1,940 degrees were awarded. The figures for $1974-75$ were 1,793 new students and 
Table 22

RATIO BETWEENT PH.D. ENROLIETT AID PH.D. ANTARDS BY SELECTED DISCIPLINES, $1969-70$ to $1975-76$

\begin{tabular}{|c|c|c|c|c|}
\hline & $\begin{array}{l}\text { Seven Years } \\
\text { TOIAL } \\
\text { ENROLMENT }\end{array}$ & $\begin{array}{l}\text { Seven year } \\
\text { TOTAL, } \\
\text { DEGREES GRANTED }\end{array}$ & PERCENVAGE & $\begin{array}{l}\text { AVERAGE ANNUAL } \\
\text { NUMBER OF } \\
\text { DEGREES GRANTED }\end{array}$ \\
\hline Classics & 596 & 54 & 9.1 & 8 \\
\hline History & 4,503 & 350 & 7.8 & 50 \\
\hline English & 5,630 & 409 & 7.3 & $5 \varepsilon$ \\
\hline French & 2,657 & .164 & 6.2 & 23 \\
\hline $\begin{array}{l}\text { Modern Languages } \\
\& \text { Literature }\end{array}$ & 2,327 & 159 & 6.8 & 23 \\
\hline Philosophy & 3,247 & 254 & 7.8 & 36 \\
\hline Religious Studies & 1,419 & 117 & 8.2 & 17 \\
\hline $\begin{array}{l}\text { Anthropology \& } \\
\text { Archaeology }\end{array}$ & 1,379 & 78 & 5.6 & 11 \\
\hline $\begin{array}{l}\text { Commerce, Business } \\
\text { Administration }\end{array}$ & 646 & 55 & 8.5 & $\varepsilon$ \\
\hline Econamics & 2,395 & 150 & 6.3 & 21 \\
\hline Geography & 1,744 & 168 & 9.6 & 24 \\
\hline Law & 459 & 45 & 9.8 & 6 \\
\hline Political science & 3,093 & 161 & 5.2 & 23 \\
\hline Psychology & $7,63 j$ & 303 & 10.5 & 115 \\
\hline Sociology & 2,572 & 141 & 5.5 & $2 C$ \\
\hline Med icine & 5,015 & 1,051 & 21.0 & 150 \\
\hline Pharmacy & 823 & 193 & 23.5 & 28 \\
\hline Mathematics & 4,479 & 664 & 14.8 & 95 \\
\hline Chemistry & 6,111 & 1,425 & 23.3 & 204 \\
\hline Geology & 1,604 & 256 & 15.1 & 37 \\
\hline Physics & 4,797 & 958 & 20.0 & 137 \\
\hline ALL DISCIPLINES* & 90,669 & 11,708 & 122.3 & 1,673 \\
\hline
\end{tabular}

Source: Derived from the Canadian Association of Graduate Schools' data.

* Includes other disciplines not identified.

1,900 Ph.D.s granted (Table 23). Consequently, taking the drop-out rate for new students into account, there will be a substantial decline in the number of degrees conferred three to five years hence. Chemistry exemplifies this boom or bust cycle. For seven years Canadian universities awarded an annual average of 204 Ph.D.s, but in $1973-74$ only 57 students enrolled; 103 in 1974-75 and 83 in 1975-76. Thus, a substantial decline in Ph.D.s is likely in three years. However, in 1975.76 the number of new doctoral students 
New Doctoral Students as a Percentage of Doctoral

Enrolment, 1973-74 to 1975-76

\begin{tabular}{|c|c|c|c|c|c|c|c|c|c|}
\hline & \multicolumn{3}{|c|}{$1973-74$} & \multicolumn{3}{|c|}{$1974-75$} & \multicolumn{3}{|c|}{$1975-76$} \\
\hline & Enrolment* & $\begin{array}{c}\text { New } \\
\text { Students }\end{array}$ & $\%$ & Enrolment* & $\begin{array}{c}\text { New } \\
\text { Students }\end{array}$ & $\%$ & Enrolment* & $\begin{array}{c}\text { New } \\
\text { Students }\end{array}$ & $\%$ \\
\hline EDUCATION & 1,205 & 139 & 11.5 & 1,298 & 145 & 11.2 & 1,335 & 264 & 19.8 \\
\hline Fine Arts & 92 & 8 & 8.7 & 92 & 3 & 3.3 & 147 & 18 & 12.2 \\
\hline Classics & 81 & 7 & 8.6 & 65 & 6 & 9.2 & 68 & 10 & 14.7 \\
\hline History & 677 & 59 & 8.7 & 657 & 71 & 10.8 & 645 & 92 & 14.3 \\
\hline English & 875 & 98 & 11.2 & 853 & 113 & 13.2 & 816 & 152 & 18.6 \\
\hline Erench & 362 & 24 & 6.6 & 344 & 34 & 9.9 & 357 & 37 & 10.4 \\
\hline Library Science & 10 & 5 & 50.0 & 7 & 1 & 14.3 & 10 & 2 & 20.0 \\
\hline $\begin{array}{l}\text { Modern Languages } \\
\text { and Literature }\end{array}$ & 442 & 30 & 6.8 & 404 & 31 & 7.7 & 400 & 46 & 11.5 \\
\hline Philosophy & 470 & 42 & 3.5 & 460 & 61 & 13.3 & 453 & 56 & 12.4 \\
\hline Religious Studies & 204 & 14 & 6.9 & 197 & 15 & 7.6 & 193 & 21 & 10.9 \\
\hline Other & 20 & 5 & 25.0 & 32 & 3 & 9.4 & 75 & 6 & 8.0 \\
\hline HUMANITIES (Total) & 3,233 & 290 & 8.4 & 3,111 & 338 & 10.9 & 3,164 & 440 & 13.9 \\
\hline $\begin{array}{l}\text { Anthropology and } \\
\text { Archaeology }\end{array}$ & 198 & 19 & 9.6 & 214 & 30 & 14.0 & 258 & 23 & 8.9 \\
\hline Area Studies & 198 & 14 & 7.1 & 210 & 29 & 13.8 & 178 & 17 & 9.6 \\
\hline $\begin{array}{l}\text { Commerce, Business } \\
\text { Administration }\end{array}$ & 99 & 9 & 9.1 & 112 & 27 & 24.1 & 114 & 29 & 25.4 \\
\hline Economics & 377 & 61 & 16.2 & 404 & 32 & 20.3 & 429 & 97 & 22.6 \\
\hline Geography & 244 & 39 & 16.0 & 250 & 33 & 13.2 & 229 & 55 & 24.0 \\
\hline Law & 60 & 2 & 3.3 & 67 & 11 & 16.4 & 63 & 7 & 11.1 \\
\hline Political Science & 486 & 74 & 15.2 & 509 & 82 & 16.1 & 522 & 78 & 14.9 \\
\hline Psychology & 1,153 & 139 & 12.1 & 1,217 & 195 & 16.0 & 1,326 & 294 & 22.2 \\
\hline Social Work & 37 & 7 & 18.9 & 40 & 5 & 12.5 & 45 & 7 & 15.6 \\
\hline Sociology & 421 & 51 & 12.1 & 421 & 61 & 14.5 & 449 & 77 & 17.1 \\
\hline Other & 50 & 9 & 18.0 & 73 & 21 & 28.8 & 0 & 26 & - \\
\hline SOCIAL SCIENCES & 3,323 & 424 & 12.8 & 3,517 & 576 & 16.4 & 3,613 & 710 & 19.7 \\
\hline
\end{tabular}

Source: Derived from the Canadian Association of Craduate Schools' data.

* Both full and part-time doctoral students. 
Table 23 (cont'd)

New Doctoral Students as a Percentage of Doctoral

Enrolment, $1973-74$ to $1975-76$

\begin{tabular}{|c|c|c|c|c|c|c|c|c|c|}
\hline & \multicolumn{3}{|c|}{$1973-74$} & \multicolumn{3}{|c|}{$1974-75$} & \multicolumn{3}{|c|}{$1975-76$} \\
\hline & Enrolment ${ }^{\hbar}$ & $\begin{array}{c}\text { New } \\
\text { Students }\end{array}$ & $\%$ & Enrolment* & $\begin{array}{c}\text { New } \\
\text { Students }\end{array}$ & 7 & Enrolment* & $\begin{array}{c}\text { New } \\
\text { Students }\end{array}$ & $\%$ \\
\hline BIOLOGICAL SCIENCES & 923 & 139 & 15.1 & 1,000 & 142 & 14.2 & 1,087 & 224 & 20.6 \\
\hline APPLIED SCIENCES & 1,263 & 150 & 11.9 & 1,158 & 132 & 11.8 & 1,267 & 165 & 13.0 \\
\hline Dentistry & 10 & 1 & 10.0 & 12 & 1 & 8.3 & 12 & 2 & 16.7 \\
\hline Medicine & 695 & 101 & 14.5 & 644 & 110 & 17.1 & 675 & 144 & 21.3 \\
\hline Pharmacy & 101 & 9 & 8.9 & 91 & 14 & 15.4 & 9]. & 25 & 27.5 \\
\hline Other & 40 & 5 & 45.5 & 27 & 6 & 22.2 & 30 & 7 & 23.3 \\
\hline HEALTH SCIENCES & 846 & 116 & 13.7 & 774 & 131 & 16.9 & 808 & 178 & 22.0 \\
\hline Mathematics & 621 & 102 & 16.4 & 571 & 85 & 14.9 & 580 & 113 & 19.5 \\
\hline Chemistry & 760 & 57 & 7.5 & 716 & 103 & 14.4 & 663 & 83 & 12.5 \\
\hline Geology & 255 & 50 & 19.6 & 260 & 45 & 17.3 & 277 & 45 & 16.2 \\
\hline Physics & 641 & 66 & 10.3 & 589 & 55 & 9.3 & 512 & 64 & 12.5 \\
\hline other & 51 & 12 & 23.5 & 67 & 36 & 53.7 & 26 & 20 & 76.9 \\
\hline PHYSICAL SCIENCES & 2,328 & 287 & 12.3 & 2,203 & 324 & 14.6 & 2,058 & 325 & 15.8 \\
\hline TOTAL & 13,121 & 1,545 & 11.8 & 13,061 & 1,793 & 13.7 & 13,332 & 2,306 & 17.3 \\
\hline
\end{tabular}

Source: Derived from the Canadian Association of Graduate Schools' data.

* Both full and part-time doctoral students. 
has increased to 2,306 or $17.3 \%$ of the total doctoral enrolment with considerable variations by discipline.

To this point, discussion has dwelt only on supply. Graduate students in chemistry, as in many other disciplines, have reacted to current diminishing employment opportunities by not continuing to the doctoral level, although information about the demand in three to five years is imperfect. This may be a wise course of action for individual students, but collectively, it creates recurring imbalances.

Universities might consider establishing ratios of the number of new Ph.D. students to the total enrolled. In 1973-74, this ratio was $11.8 \%, 13.7 \%$ in $1975-76$ for all fields, but varied among disciplines. The problem is to determine the ideal ratio, taking both supply and demand into consideration. The next section simulates anticipated supply and demand for Ph.D.s for university teaching from 1977-78 to 1981-82.

\section{PH.D. SUPPLY AND DEMAND IN THE UNIVERSITY SECTOR}

The demand for Ph.D.s in the next five years is difficult to predict. It has been estimated that only $1.3 \%$ to $1.5 \%$ of the present 35,000 Ph.D. positions will have to be replaced each year - fewer than 500 annually for the next few years. This means that one out of four of the 2,000 new Ph.D.s produced each year will be absorbed as replacements for Ph.D. holders who retire, die, or withdraw for health reasons.

Historically, education and government have employed about $85 \%$ of the Ph.D.s in Canada. The present economic climate indicates that those two sectors will utilize a much reduced number of Ph.D.s. Austerity measures instituted by the federal and provincial governments will decrease employment opportunities in the public sector. The combination of financial constraints and demographic trends have the same effect on university teaching positions. Demographic patterns indicate that in a few years, the source population for post-secondary students (18-24 years old) will drop from 3.3 million to 2.7 million, and universities will have to anticipate a decline in enrolment, provided that the participation rate for post-secondary education does not change markedly.

A model, described in Appendices A and $\mathrm{B},{ }^{*}$ simulates the supply and demand for Ph.D.s in universities. After adjustments for other employment possibilities the balance is considered a potential surplus.

The model treats each of the 42 discipline categories separately, and assumes that there is no substitutability among them. For example, a deficit in dentistry cannot be filled by a surplus in pharmacy. Tables 24 and 25 summarize the supply and demand pattern of Ph.D.s by discipline for university teaching, and serve as a basis for a five-year projection.

For most disciplines, the surplus is small in absolute numbers, but large in percentage terms. For example, there is a supply of 12 Ph.D.s in classics and a demand for 6 , creating a surplus of 6 persons, but this means $50.0 \%$ under-utilization.

Between 1977-78 and 1981-82 there will be a cumulative surplus of 3,230 Ph.D.s, 1,780 in the natural sciences and 1,250 in the humanities and social sciences (Table 26). Only in the health sciences do supply and demand seem to balance more. Nevertheless,

\footnotetext{
* Copies of the Appendices are available from the Editor.
} 
Table 24

Supply of Ph.D.s for University Teaching by Discipline, 1976-77

\begin{tabular}{|c|c|c|c|c|c|c|}
\hline \multirow{2}{*}{$\begin{array}{c}\mathrm{Ph.D} . \\
\text { enrolment }\end{array}$} & \multicolumn{2}{|c|}{ Less Foreign Students } & \multicolumn{2}{|c|}{ Withdrawal rate } & \multicolumn{2}{|c|}{$\begin{array}{l}\text { Length of. Study } \\
\text { until graduation }\end{array}$} \\
\hline & in percent & balance & in percent & balance & years & $\begin{array}{c}\text { Degree } \\
\text { granted } \\
\text { each year }\end{array}$ \\
\hline
\end{tabular}

Humanities

Fine \& Applied Arts

Classics

istory

English

French

other Modern Languages

and linguistics

Philosophy

Religious Studies

Others

Sub-total Humanities

Social Sciences

Anthropology

Archaeology

Area Studies

Business Administration

Economics

ducation

Geography

Law

Political Science

Psychology

Social Work

Sociology

Other

Sub-total Social Sciences

Applied Sciences

Architecture

Chemical Engineering

Civil Engineering

Electrical Engineering

$\begin{array}{rrr}147 & 5 & 140 \\ 68 & 10 & 61 \\ 605 & 10 & 544 \\ 816 & 10 & 734 \\ 357 & 10 & 321 \\ 472 & 10 & 431 \\ 453 & 10 & 408 \\ 193 & 10 & 174 \\ 50 & 10 & 45 \\ 3,161 & & 2,858\end{array}$

60

$40-33$

$55-330$

$55 \quad 144$

$60 \quad 172$

$45 \quad 224$

45

45

218

40

210

114

429

, 335

229

63
522

32

45

449

113

5,093

$\begin{array}{rr}10 & 196 \\ 10 & 36 \\ 10 & 189 \\ 10 & 103 \\ 10 & 386 \\ 5 & 1,268 \\ 10 & 206 \\ 10 & 57 \\ 10 & 470 \\ 10 & 1,193 \\ 10 & 41 \\ 10 & 404 \\ 10 & 102 \\ & 4,651\end{array}$

$\begin{array}{rrrr}50 & 98 & 5 & 20 \\ 50 & 18 & 5 & 4 \\ 50 & 94 & 5 & 19 \\ 50 & 51 & 5 & 10 \\ 50 & 143 & 5 & 39 \\ 60 & 507 & 5 & 101 \\ 40 & 124 & 5 & 25 \\ 50 & 29 & 5 & 6 \\ 65 & 164 & 5 & 33 \\ 45 & 656 & 5 & 131 \\ 50 & 21 & 5 & 4 \\ 65 & 141 & 5 & 28 \\ 50 & 51 & 5 & 10 \\ & 2,147 & 5 & 430\end{array}$

$\begin{array}{rrr}41 & 15 & 37 \\ 187 & 15 & 159 \\ 195 & 15 & 166 \\ 322 & 15 & 274\end{array}$

$\begin{array}{rr}50 & 17 \\ 15 & 135 \\ 15 & 141 \\ 15 & 233\end{array}$


Table 24

Supply of Ph.D.s for University Teaching by Discipline, 1976-77 (cont'd)

Mechanical Engineering

Mining

Forestry

other

Sub-Total Applied Sciences

\begin{tabular}{|c|c|c|c|c|c|c|}
\hline \multirow{2}{*}{$\begin{array}{l}\text { Ph.D. } \\
\text { enrolment }\end{array}$} & \multicolumn{2}{|c|}{ Less Foreign Students } & \multicolumn{2}{|c|}{ Withdrawal rate } & \multicolumn{2}{|c|}{$\begin{array}{l}\text { Length of Study } \\
\text { until graduation }\end{array}$} \\
\hline & in percent & balance & in percent & balance & years & $\begin{array}{c}\text { Degree } \\
\text { granted } \\
\text { each year }\end{array}$ \\
\hline 177 & 15 & 150 & 15 & 128 & 3 & 43 \\
\hline 111 & 15 & 94 & 15 & 80 & 3 & 27 \\
\hline 54 & 15 & 46 & 40 & 28 & 3 & 9 \\
\hline 134 & 15 & 114 & 50 & 57 & 3 & 19 \\
\hline 1,221 & & 1,040 & & 819 & 3 & 274 \\
\hline
\end{tabular}

Biological Sciences

Agriculture

Biology

Botany

Veterinary Medicine

Zoology

other

Sub-Total Biological Sciences

$\begin{array}{rlr}94 & 10 & 85 \\ 310 & 10 & 279 \\ 200 & 10 & 180 \\ 74 & 10 & 67 \\ 240 & 10 & 216 \\ 70 & 10 & 63 \\ 983 & & 890\end{array}$

$\begin{array}{rrrr}20 & 68 & 3 & 23 \\ 50 & 139 & 3 & 46 \\ 10 & 162 & 3 & 54 \\ 20 & 54 & 3 & 18 \\ 20 & 173 & 3 & 58 \\ 20 & 50 & 3 & 17 \\ & 646 & 3 & 216\end{array}$

Health Sciences

Dentistry

Medicine

Pharmacy

Other

Sub-Total Health Sciences

Physical Sciences Mathematics and related

Chemistry

Geology

Physics

ther

Sub-Total Physical Sciences

TO'TAL

$\begin{array}{rrr}12 & 10 & 11 \\ 668 & 10 & 601 \\ 91 & 10 & 82 \\ 40 & 10 & 36 \\ 811 & & 730 \\ & & \\ 570 & 15 & 484 \\ 663 & 15 & 564 \\ 277 & 15 & 235 \\ 512 & 15 & 435 \\ 36 & 15 & 31 \\ 2,058 & & 1,749 \\ & & 11,918\end{array}$

$\begin{array}{rrrr}20 & 9 & 3 & 3 \\ 25 & 451 & 3 & 150 \\ 20 & 66 & 3 & 22 \\ 20 & 29 & 3 & 10 \\ & 555 & 3 & 185\end{array}$

$\begin{array}{rrrr}35 & 315 & 3 & 105 \\ 15 & 479 & 3 & 160 \\ 50 & 117 & 3 & 39 \\ 30 & 305 & 3 & 102 \\ 50 & 15 & 3 & 5 \\ & 1,231 & 3 & 411 \\ & 6,814 & & 1,799\end{array}$




\begin{tabular}{|c|c|c|c|c|c|c|c|c|}
\hline \multicolumn{3}{|c|}{ Returning Canadians } & \multirow{2}{*}{$\begin{array}{l}\text { Sub- } \\
\text { Total }\end{array}$} & \multirow{2}{*}{$\begin{array}{c}\text { Total } \\
\text { Canadian } \\
\text { and Foreign }\end{array}$} & \multicolumn{2}{|c|}{ Less already employed } & \multicolumn{2}{|c|}{$\begin{array}{c}\text { University } \\
\text { teaching }\end{array}$} \\
\hline United States & United Kingdom & $\begin{array}{c}\text { other } \\
\text { countries }\end{array}$ & & & in percent & balance & in percent & balance \\
\hline
\end{tabular}

Humanities

Fine \& Applied Arts

Classics

History

English

French

Other Modern Languages

and linguistics

Philosophy

Religious Studies

Others

Sub-Total Humanities

$\begin{array}{rr}5 & 2 \\ 2 & 3 \\ 8 & 4 \\ 6 & 5 \\ 2 & 1 \\ 3 & 1 \\ 5 & 6 \\ 1 & 1 \\ 2 & 1 \\ 34 & 24\end{array}$

Social Sciences

Anthropology

Archaeology

Area Studies

Business Administration

Economics

Education

Geography

Law

Political Science

Psychology

Soclal Work

Sociology

Other

Sub-Total Social Sciences

4
1
4
2
4
4
8
2
1
30

$\begin{array}{rr}11 & 22 \\ 6 & 15 \\ 16 & 81 \\ 13 & 79 \\ 7 & 36 \\ 8 & 42 \\ 19 & 64 \\ 4 & 23 \\ 4 & 9 \\ 88 & 371\end{array}$

15
15
15
15
15
15
15
15
15
15

$\begin{array}{rrr}19 & 90 & 17 \\ 13 & 90 & 12 \\ 69 & 90 & 62 \\ 67 & 90 & 60 \\ 31 & 90 & 28 \\ 36 & 90 & 32 \\ 54 & 90 & 49 \\ 20 & 90 & 18 \\ 8 & 90 & 7 \\ 317 & & 285\end{array}$

Applied Sciences

Architecture

Chemical Engineering

Civil Engineering

Electrical Engineering

3
3
9
10
15
3
2
5
15
1
5
3
74

$\begin{array}{rr}20 & 20 \\ 7 & 11 \\ 6 & 25 \\ 9 & 1 \\ 19 & 58 \\ 20 & 12 \\ 8 & 33 \\ 5 & 11 \\ 16 & 49 \\ 20 & 15 \\ 3 & \\ 13 & 4 \\ 5 & 15 \\ 131 & 56\end{array}$

$\begin{array}{rrrr}15 & 17 & 75 & 13 \\ 15 & 9 & 75 & 8 \\ 15 & 21 & 75 & 16 \\ 15 & 16 & 75 & 12 \\ 15 & 47 & 50 & 24 \\ 15 & 103 & 50 & 52 \\ 15 & 28 & 75 & 21 \\ 15 & 9 & 50 & 4 \\ 15 & 42 & 75 & 32 \\ 15 & 128 & 50 & 64 \\ 15 & 6 & 75 & 4 \\ 15 & 35 & 75 & 26 \\ 15 & 13 & 75 & 10 \\ & 474 & & 286\end{array}$

13
8
16
12
24
52
21
4
32
64
4
26
10
286

$\begin{array}{ll}1 & 2 \\ 3 & 3 \\ 3 & 3 \\ 5 & 5\end{array}$

$\begin{array}{rr}6 & 12 \\ 6 & 5 \\ 6 & 5 \\ 11 & 8\end{array}$

12
51
53
89

5
5
5
5

$\begin{array}{ll}11 & 50 \\ 48 & 50 \\ 50 & 50 \\ 85 & 50\end{array}$

6
24
25
42 
Table 24

Supply of Ph.D.s for University Teaching by Discipline, 1976-77 (cont'd)

Mechanical Engineering

Mining

Forestry

Other

Sub-Total Applied Sciences

\begin{tabular}{|c|c|c|}
\hline \multicolumn{2}{|c}{ Returning Canadians } \\
\hline United States & United Kingdom & $\begin{array}{r}\text { ot } \\
\text { count }\end{array}$ \\
\hline 3 & 3 \\
3 & 2 \\
1 & 1 \\
2 & 3 \\
21 & 22
\end{tabular}

Biological Sciences Agriculture

Biology

Botany

Veterinary Medicine

Zoology

Sub-Total Biological Sciences

6
5
8
3
8
2
32

1

Health Sciences

Dentistry

Medicine

Pharmacy

Other

Sub-Total Health Sciences

Physical Sciences Mathematics

and related

Chemistry

Geology

Physics

Other

Sub-Total Physical Sciences

TOTAL

\begin{tabular}{|r} 
Sub- \\
Tota \\
\hline \\
6 \\
5 \\
3 \\
8 \\
51
\end{tabular}

\begin{tabular}{|c|c|c|c|c|}
\hline \multirow{2}{*}{$\begin{array}{c}\text { Total } \\
\text { Canadian } \\
\text { and Foreion }\end{array}$} & \multicolumn{2}{|c|}{ Less already employed } & \multicolumn{2}{|c|}{$\begin{array}{c}\text { University } \\
\text { teaching }\end{array}$} \\
\hline & in percent & balance & in percent & balance \\
\hline
\end{tabular}

325

$\begin{array}{rrrr}5 & 47 & 50 & 24 \\ 5 & 30 & 50 & 15 \\ 5 & 11 & 50 & 6 \\ 5 & 26 & 50 & 13 \\ & 308 & & 155\end{array}$

$\begin{array}{rrrrr}31 & 5 & 29 & 50 & 14 \\ 55 & 5 & 52 & 50 & 26 \\ 66 & 5 & 63 & 50 & 32 \\ 23 & 5 & 22 & 50 & 11 \\ 70 & 5 & 66 & 50 & 33 \\ 23 & 5 & 22 & 50 & 11 \\ 268 & & 254 & & 127\end{array}$

1
1
1
0
3

1
1
1
0
3
2
5
2
10
2
21

6
159
26
12
203

121
180
52
131
14
498

5
5
5
5

6
151
25
11
193

$\begin{array}{rr}50 & 3 \\ 50 & 76 \\ 50 & 12 \\ 50 & 6 \\ & 97\end{array}$

10

$\begin{array}{rr}5 & 115 \\ 5 & 171 \\ 5 & 49 \\ 5 & 124 \\ 5 & 13 \\ & 472 \\ & \end{array}$

$\begin{array}{rr}50 & 58 \\ 40 & 86 \\ 50 & 24 \\ 50 & 62 \\ 50 & 6 \\ & 236 \\ & \end{array}$


Table 25

Demand and Supply of University Teachers with a Doctorate Degree, 1977-78

Education

Fine and Applied Arts

Classics

History

English

French

Other Modern Languages

Philosophy

Religious Studies

Sub-Total Humanities

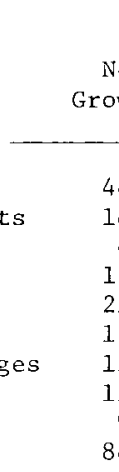

Anthropology

Area Studies

Commerce, Business

Administration

Economics

Geography

Law

Political Science

Psychology

Social Work

Sociology

Sub-Total Social Sciences

TOTAL HUMAN SCIENCES

Agriculture

Biology

Botany

Veterinary Medicine

Zoology

Sub-Total Biological

Sciences

Proportion

Effective Potential

Surplus (+) Percentage Demand $(-)$ under-utilized

Towal

Doctorate

Efrectivi

23.1

50.0

66.1

53.3

42.8

34.4

1.4

33.

54.8

+
$+\quad 6$
+143

$+143$

66.7

$+14 \quad 87.5$

$-10$

$+8$

$+10$

$-6$

$+19$

$+38$

$+\overline{10}$

+10
$+\quad 97$

97
250

47.6

59.4

59.4

38.5

50.0

+7
+

$+12$

$+28$

$+\quad 7$
+

46.2
87.5

87.5
63.6

11
33

+25
$+\quad 7$

75.8

$-$

$\begin{array}{r}+79 \\ \hline\end{array}$

64.6 
Table 25

Demand and Supply of University Teachers with a Doctorate Degree, 1977-78 (cont'd)

Architecture
Chemical Engineering
Civil Engineering
Electrical Engineering
Mechanical Engineering
Mining Engineering
Forestry
Other Applied Sciences
ub-Total Applied Sciences

$\begin{array}{cc}\text { New } & \text { New } \\ \text { Growth } & \text { Peplacement } \\ \text { Total }\end{array}$

Proportion
with

Effective

Demand

Potential
Supply

Supply (t) Percentage

- $2 . .750$

-

\section{Dentistry \\ Medicine \\ Pharmacy}

Sub-Total Health

$$
\text { Professions }
$$

3
4
6
6
5
2
2
6
34

Mathematics and related

Chemistry

Geology and Related

Physics

Sub-Total Physical

Sciences

$\begin{array}{rr}2 & 5 \\ 2 & 6 \\ 4 & 10 \\ 4 & 10 \\ 3 & 8 \\ 1 & 3 \\ 2 & 4 \\ 4 & 10 \\ 22 & 56\end{array}$

50
75
75
75
75
75
75
75
-

$\begin{array}{rrr}3 & 7 & 75\end{array}$

30

45
2

75

75
85

$\begin{array}{llll}51 & 35 & 86 & -\end{array}$

$-$

(2)

$19 \quad 13$

$13 \quad 32$

1128

75
90

15

8
22

66

44

13
37

37
110

90

90
90

TOTAL NATURAL SCIENCES 179

120

299

$-$

GRAND TOTAL

$446 \quad 300$

746

2
4
8
8
6
2
3
8

6
24
25
42
24
15
6
13
155

-

66.7

83.3

68.0

33.3

86.

56.7

50.0

73.5

26.3

75.0

29.7

58.6

70.9

46.8

59.1

59.4

* Grand total includes demand and supply information for the "other" disciplines not identified. 
Table 26

Supply and Demand of University Teachers with a Ph.D. Degree by Teaching Field, 1977-78 to 1981-82

\begin{tabular}{|c|c|c|c|c|c|c|c|c|c|c|c|c|c|c|c|}
\hline \multirow[b]{3}{*}{ Humanities } & \multicolumn{3}{|c|}{$1977-78$} & \multicolumn{3}{|c|}{$1978-79$} & \multicolumn{3}{|c|}{$1979-80$} & \multicolumn{3}{|c|}{$1980-81$} & \multicolumn{3}{|c|}{$1981-82$} \\
\hline & \multirow{2}{*}{$\begin{array}{l}\text { Supply } \\
278\end{array}$} & \multicolumn{2}{|c|}{$\begin{array}{l}\text { Surplus (+) } \\
\text { Demand or } \\
\text { Deficit }(-)\end{array}$} & \multirow{2}{*}{$\frac{\operatorname{Supply}}{278}$} & \multicolumn{2}{|c|}{$\begin{array}{c}\text { Surplus (t) } \\
\text { Demand or } \\
\text { Deficit (-) }\end{array}$} & \multirow{2}{*}{$\frac{\mid \text { Supoly }}{278}$} & \multicolumn{2}{|c|}{$\begin{array}{c}\text { Surplus }(+) \\
\text { Demand or } \\
\text { Deficit }(-)\end{array}$} & \multirow{2}{*}{$\frac{\mid \text { Supply }}{278}$} & \multicolumn{2}{|c|}{$\begin{array}{c}\text { Surplus ( }+) \\
\text { Demand or } \\
\text { Deficit (-) }\end{array}$} & \multirow{2}{*}{$\underbrace{\int \text { Supply }}_{278}$} & \multicolumn{2}{|c|}{$\begin{array}{l}\text { Surplus (+) } \\
\text { Demand or } \\
\text { Deficit (-) }\end{array}$} \\
\hline & & 137 & +141 & & 137 & +141 & & 137 & +141 & & 137 & +141 & & 137 & +141 \\
\hline Social Sciences & s 284 & 175 & +109 & 284 & 175 & +109 & 284 & 175 & +109 & 284 & 175 & +109 & 284 & 175 & +109 \\
\hline $\begin{array}{l}\text { Sub-Total } \\
\text { Human Sciences }\end{array}$ & 562 & 312 & +250 & 562 & 312 & +250 & 562 & 312 & +250 & 562 & 312 & +250 & 562 & 312 & +250 \\
\hline $\begin{array}{l}\text { Applied } \\
\text { Sciences }\end{array}$ & 155 & 41 & +114 & 155 & 41 & +114 & 155 & 41 & +114 & 155 & 41 & +114 & 155 & 41 & +114 \\
\hline $\begin{array}{l}\text { Biological } \\
\text { Sciences }\end{array}$ & 116 & 37 & +79 & 116 & 37 & +79 & 116 & 37 & +79 & 116 & 37 & +79 & 116 & 37 & +79 \\
\hline Health Sciences & s 91 & 64 & +27 & 91 & 64 & +27 & 91 & 64 & +27 & 91 & 64 & +27 & 91 & 64 & +27 \\
\hline $\begin{array}{l}\text { Physical } \\
\text { Sciences }\end{array}$ & 230 & 94 & +136 & 230 & 94 & +136 & 230 & 94 & +136 & 230 & 94 & +136 & 230 & 94 & +136 \\
\hline $\begin{array}{l}\text { Sub-Total } \\
\text { Natural Scs. }\end{array}$ & 592 & 236 & +356 & 592 & 236 & +356 & 592 & 236 & +356 & 592 & 236 & +356 & 592 & 236 & +356 \\
\hline GRAND TOTAL & 1,194 & 548 & +646 & 1,194 & 548 & +646 & 1,194 & 548 & +646 & 1,194 & 548 & +646 & 1,194 & 548 & +646 \\
\hline
\end{tabular}


if some of the assumptions underlying the model change, the situation could be different. For example, universities simply might not hire new faculty although an increase in enrolment over the next few years is likely. Or, to economize, they might fill positions that become vacant through retirement and death with graduate students and part-time teachers. This is appealing for universities whose financial resources have been reduced in relative terms. It is even more attractive in view of the fact that as they acquire seniority, faculty move into higher ranks with higher salaries, compared to those of lecturers and assistant professors. As another economy measure, provincial governments and universities might consider a slight increase in the student-teacher ratio which would mean a substantial saving of positions each year.

The future prospect is that few teachers will be employed in relation to the total. This could have serious implications for the quality of university education in Canada. During the sixties, universities had to rely on less than fully-trained personnel to meet the growing demand, many of whom are still employed. Now when there is an adequate supply of Ph.D.s, even the best experience difficulty obtaining university positions. Without suitable employment it is difficult for them to keep abreast of research in their field, and there is a danger that their training may become obsolete.

The supply of Ph.D.s, at least for the next five years, can be projected more accurately because doctoral students now enrolled will still be in the system. Nevertheless, particularly at the discipline level, the figures are meant to indicate the magnitude of the problem rather than to predict precise numerical values. From a policy point of view this exercise should be regarded as only one type of analysis, which needs to be supplemented by information from other sources and judgmental considerations.

This simulation seems to indicate that the employment opportunities in the university sector for the next five years will be limited and many Ph.D. holders will have to pursue other career alternatives. This scenario does not only provide challenges for the individual Ph.D. recipient, but also to the universities as well as government and industry. They will have to develop new avenues of employment for this group of highly-skilled and motivated young Ph.D. holders. ${ }^{7}$

\section{List of Tables}

Table No.

1. Employment of Ph.Ds by Industrial Sector and by Age, 1973

2. Selected Occupations of Ph.Ds by Sex, 1973

3. Geographic Origin of Ph.Ds, 1973

4. Foreign born Ph.Ds by Country of Birth and Period of Immigration, 1973

5. Full-Time University Teachers by Field of Study, 1956-57 to 1974-75

6. Immigrants to Canada by Country of Last Permanent Residence and Intended Occupation: "University Teaching", 1962 to 1974

${ }^{7}$ It needs to be stressed that these figures do not indicate the actual magnitude of unemployment of Ph.D. holders. It means that doctoral graduates will have to look beyond the traditional university employment sector. It seems unlikely that Ph.D.s will be unemployed, but a certain amount of under-employment may be expected and that Ph.D. holders may displace those who are less qualified. 
7. Characteristics of University Teachers by Discipline, 1973-74

8. Employment of Ph.Ds by Year of Appointment and Discipline in Federal Departments under the Public Service Employment Act, 1940 to 1972

9. Employment Sector of Ph.Ds Immediately after Graduation from Canadian Universities by Field of Study, 1970-71 to 1974-75

10. Doctoral Degrees Awarded by Field of Study, 1960-61 to 1973.74

11. Doctoral Degrees Awarded by Selected Disciplines, 1960-61 to 1972-73

12. Full and Part-Time Doctoral Student Enrolment by Field of Study, 1969-70 to $1975-76$

13. Full and Part-Time Doctoral Enrolment at Five Selected Universities, 1968-69 to 1975.76

14. Citizenship and Immigration Status of Full-Time Masters and Ph.D. Students by Field of Study, 1972-73

15. Citizenship of Full-Time Ph.D. Students by Country and Field of Study, 1972.73

16. Canada Council Doctoral Fellowship Holders by Discipline, 1965-66 to 1974-75.

17. Canada Student Loan Plan Certificates for Doctoral Students by Province, 1964-65 to $1974-75$.

18. Canada Student Loan Plan Doctoral Student Recipients by Province or Country of Study, 1964-65 to 1974-75.

19. Number of Canadian Universities Offering Masters and Doctoral Degree Programs, 1944-45 to 1974-75.

20. Number of Doctoral Programs at Canadian Universities by Discipline, 1974-75.

21. Ratio between Ph.D. Enrolment and Ph.D. Awards by Field of Study, 1969-70 to 1974-75.

22. Ratio between Ph.D. Enrolment and Ph.D. Awards by Selected Discipline, 1969-70 to $1975-76$.

23. New Doctoral Students as a percentage of Doctoral Enrolment, 1973-74 to 1975-76.

24. Supply of Ph.Ds for University Teaching by Discipline, 1976-77.

25. Demand and Supply of University Teachers with a Doctorate Degree by Discipline, 1977-78.

26. Supply and Demand of University Teachers with a Ph.D. degree by Field of Study, $1977-78$ to $1981-82$. 